RUP-17-12, KEK-Cosmo-206, KEK-TH-1987, OCU-PHYS-466, AP-GR-139

\title{
Spins of primordial black holes formed in the matter-dominated phase of the Universe
}

\author{
Tomohiro Harada* \\ Department of Physics, Rikkyo University, Toshima, Tokyo 171-8501, Japan \\ ${ }^{1}$ Chul-Moon Yoo \\ ${ }^{1}$ Gravity and Particle Cosmology Group, \\ Division of Particle and Astrophysical Science, \\ Graduate School of Science, Nagoya University, Nagoya 464-8602, Japan \\ ${ }^{2,3,4}$ Kazunori Kohri \\ ${ }^{2}$ Institute of Particle and Nuclear Studies, KEK, \\ 1-1 Oho, Tsukuba, Ibaraki 305-0801, Japan \\ ${ }^{3}$ The Graduate University for Advanced Studies (SOKENDAI), \\ 1-1 Oho, Tsukuba, Ibaraki 305-0801, Japan and \\ ${ }^{4}$ Rudolf Peierls Centre for Theoretical Physics, \\ The University of Oxford, 1 Keble Road, Oxford, OX1 3NP, UK \\ ${ }^{5}$ Ken-Ichi Nakao \\ ${ }^{5}$ Department of Mathematics and Physics, \\ Graduate School of Science, Osaka City University, \\ 3-3-138 Sugimoto, Sumiyoshi, Osaka 558-8585, Japan
}

(Dated: March 20, 2019) 


\begin{abstract}
Angular momentum plays very important roles in the formation of primordial black holes in the matter-dominated phase of the Universe if it lasts sufficiently long. In fact, most collapsing masses are bounced back due to centrifugal force, since angular momentum significantly grows before collapse. For masses with $q \leq q_{c} \simeq 2.4 \mathcal{I}^{1 / 3} \sigma_{H}^{1 / 3}$, where $q$ is a nondimensional parameter of initial reduced quadrupole moment, $\sigma_{H}$ is the density fluctuation at horizon entry $t=t_{H}$, and $\mathcal{I}$ is a parameter of the order of unity, angular momentum gives a suppression factor $\sim$ $\exp \left(-0.15 \mathcal{I}^{4 / 3} \sigma_{H}^{-2 / 3}\right)$ to the production rate. As for masses with $q>q_{c}$, the suppression factor is even stronger as $\sim \exp \left(-0.0046 q^{4} / \sigma_{H}^{2}\right)$. We derive the spin distribution of primordial black holes and find that most of the primordial black holes are rapidly rotating near the extreme value $a_{*}=1$, where $a_{*}$ is the nondimensional Kerr parameter at their formation. The smaller $\sigma_{H}$ is, the stronger the tendency towards the extreme rotation. Combining this result with the effect of anisotropy, we numerically and semianalytically estimate the production rate $\beta_{0}$ of primordial black holes. Then we find that $\beta_{0} \simeq 1.9 \times 10^{-7} f_{q}\left(q_{c}\right) \mathcal{I}^{6} \sigma_{H}^{2} \exp \left(-0.15 \mathcal{I}^{4 / 3} \sigma_{H}^{-2 / 3}\right)$ for $\sigma_{H} \lesssim 0.005$, while $\beta_{0} \simeq 0.05556 \sigma_{H}^{5}$ for $0.005 \lesssim \sigma_{H} \lesssim 0.2$, where $f_{q}\left(q_{c}\right)$ is the fraction of masses whose $q$ is smaller than $q_{c}$ and we assume $f_{q}\left(q_{c}\right)$ is not too small. We argue that matter domination significantly enhances the production of primordial black holes despite the suppression factor. If the end time $t_{\text {end }}$ of the matter-dominated phase satisfies $t_{\text {end }} \lesssim\left(0.4 \mathcal{I} \sigma_{H}\right)^{-1} t_{H}$, the effect of the finite duration significantly suppresses primordial black hole formation and weakens the tendency towards large spins.
\end{abstract}

PACS numbers: 04.70.Bw, 98.80.-k, 97.60.Lf

\footnotetext{
* harada@rikkyo.ac.jp
} 


\section{CONTENTS}

I. Introduction $\quad 4$

II. Preliminaries $\quad 5$

A. Basic equations $\quad 5$

$\begin{array}{ll}\text { B. Linear perturbations } & 7\end{array}$

$\begin{array}{lr}\text { III. Angular momentum } & 7\end{array}$

A. Second-order contribution $\quad 8$

B. First-order contribution 11

IV. Application to primordial black holes 13

A. Average angular momentum of masses 13

B. Hypothesis 14

$\begin{array}{ll}\text { C. Suppression to primordial black hole production } & 16\end{array}$

$\begin{array}{ll}\text { D. Distribution of spins of primordial black holes } & 18\end{array}$

$\begin{array}{ll}\text { V. Production rate } & 20\end{array}$

A. Production rate in the matter-dominated era 20

B. Black hole threshold in the radiation-dominated era 23

C. Comparison between the matter-dominated and radiation-dominated eras 26

$\begin{array}{ll}\text { VI. Conclusion } & 27\end{array}$

$\begin{array}{ll}\text { Acknowledgments } & 28\end{array}$

A. Exact expression for the first-order contribution in an ellipsoid 28

B. Condition for the end time in terms of the reheating temperature 29

C. Derivation of the semianalytic formula 30

$\begin{array}{ll}\text { References } & 32\end{array}$ 


\section{INTRODUCTION}

Primordial black holes may have been formed in the early Universe. Their masses are given by $M \sim\left(c^{3} / G\right) t \simeq 10^{15}\left(t / 10^{-23} \mathrm{~s}\right) \mathrm{g}$, where $t$ is the cosmological time of the formation. They have left observable signatures in the Universe until now. Observational constraints on the abundance of primordial black holes by a variety of observations are reviewed in $[1,2]$. There still remains a possibility for primordial black holes to be a large fraction of dark matter [2, 3]. Sasaki et al. [4] pointed out that binary primordial black holes can be a source of gravitational wave event GW150914 observed by LIGO [5]. See, also, Refs. [6-8] for other estimates of the merger rate. This possibility was also discussed for the recently published event GW170104 [9]. Pani and Loeb [10] discussed the imprint of superradiant instabilities of spinning primordial black holes on the spectrum of cosmic microwave background. Chiba and Yokoyama [11] obtained the spin distribution of primordial black holes and concluded that primordial black holes are mostly slowly rotating based on the critical phenomena in the collapse of rotating radiation fluid [12].

The primordial black hole formation process was pioneered in the radiation-dominated phase of the Universe by Carr [13]. In this phase, there is a threshold $\delta_{\text {th }}$ of black hole formation, which is governed by pressure gradient force, and the production rate of black holes is given by $\sim\left(\delta_{\mathrm{th}} / \sigma_{H}\right) \exp \left[-\delta_{\mathrm{th}}^{2} /\left(2 \sigma_{H}^{2}\right)\right]$, where $\sigma_{H}$ is density fluctuation at horizon entry. The threshold $\delta_{\text {th }}$ of density perturbation was originally estimated to $\sim 1 / 3[13]$ and recently to $\sim 0.42-0.56$ for relatively gentler profiles of density field [14-20]. On the other hand, black hole formation in a matter-dominated phase is not yet studied so much. A matter-dominated phase is considered not only after the matter-radiation equality but also in an earlier stage of the Universe, such as the inflaton-oscillating phase after inflation [2124] and the epoch of strong phase transition [25-27], for which the mass of the formed black holes is given in terms of the cosmological time of the epoch.

It has been conventionally believed that primordial black holes are effectively produced in the matter-dominated phase due to the absence or significant reduction of the pressure gradient force. The theory of black hole formation in the matter-dominated era was pioneered by Khlopov and Polnarev [25, 26]. It is deviation from spherical symmetry that governs the

probability of black hole formation in this phase unlike in the radiation-dominated phase. In the absence of the pressure gradient force, anisotropy develops during collapse so that 
the final stage can be described as pancake collapse [28, 29]. Harada et al. [30] reanalyzed this problem and found that the application of the hoop conjecture for black hole formation results in the production rate $\beta_{0} \simeq 0.05556 \sigma_{H}^{5}$ for $\sigma_{H} \ll 1$. Based on this estimate of the production rate, Carr et al. [31] discussed the inflaton and spectator field perturbations. It should be noted that the nonspherical effect in primordial black hole formation was also discussed by Kühnel and Sandstad [32] in a very different way.

The effect of rotation has not yet been seriously studied in the formation of primordial black holes. At first sight, it seems negligible because the rotational mode is not growing in the linear order in cosmological perturbation theory. Even in full nonlinearity, circulation is conserved in the dynamics of perfect fluid. In the current paper, however, we show that this expectation is not correct. We adopt the theory of angular momentum in structure formation, which has been developed to explain the origin of the angular momentum of galaxies by Peebles [33] and Catelan and Theuns [34]. We find that angular momentum plays very important roles in the formation of primordial black holes in the matter-dominated phase.

This paper is organized as follows. In Sec. II, we present basic equations and review cosmological perturbation theory in Newtonian gravity. In Sec. III, we introduce the angular momentum of masses and review the first-order and second-order contributions. In Sec. IV, we apply this theory to primordial black hole formation in the matter-dominated era. We derive the suppression factors of the production rate and the initial spin distribution of primordial black holes. In Sec. V, we obtain the production rate of primordial black holes in the matter-dominated era and discuss it in comparison with that in the radiation-dominated era. Section VI is devoted to conclusions.

\section{PRELIMINARIES}

\section{A. Basic equations}

We briefly review standard cosmological perturbation theory in Newtonian gravity. See e.g. Peebles [33, 35] and Hwang et al. [36] for details. We begin with the Euler equation, the equation of continuity, and the Poisson equation:

$$
\mathbf{a}=-\nabla_{r} \Psi, \quad\left(\frac{\partial \rho}{\partial t}\right)_{r}+\nabla_{r}(\rho \cdot \mathbf{v})=0, \quad \text { and } \quad \nabla_{r}^{2} \Psi=4 \pi G \rho,
$$


respectively, where $\mathbf{v}:=D \mathbf{r} / D t$ and $\mathbf{a}:=D \mathbf{v} / D t, \mathbf{r}$ is the Eulerian coordinates, $\nabla_{r}$ is the nabla with respect to $\mathbf{r}$ and $D / D$ t denotes the time derivative along the motion of a fluid element. We introduce the comoving coordinates $\mathbf{x}$, peculiar velocity $\mathbf{u}$, density perturbation $\delta$, and potential perturbation $\psi$ such that $\mathbf{x}:=\mathbf{r} / a, \mathbf{u}:=a D \mathbf{x} / D t, \delta:=\left(\rho-\rho_{0}\right) / \rho_{0}$, and $\psi:=\Psi-\Psi_{0}$, where $\rho_{0}=\rho_{0}(t)$ and $a=a(t)$ are the density and scale factor of the homogeneous and isotropic universe, respectively. Noting

$$
\frac{D f}{D t}=\frac{\partial f}{\partial t}+\frac{D \mathbf{x}}{D t} \cdot \nabla f
$$

where $\nabla$ is the nabla with respect to $\mathbf{x}$, we find

$$
\mathbf{v}=H a \mathbf{x}+\mathbf{u} \quad \text { and } \quad \mathbf{a}=\frac{\partial \mathbf{u}}{\partial t}+H \mathbf{u}+\frac{1}{a}(\mathbf{u} \cdot \nabla) \mathbf{u}+\ddot{a} \mathbf{x}
$$

where the dot denotes the derivative with respect to $t$ and $H:=\dot{a} / a$ is the Hubble parameter.

As a zeroth-order solution, we find

$$
\begin{aligned}
\rho_{0} a^{3} & =\text { const. } \\
\Psi_{0} & =\frac{2}{3} \pi G \rho_{0} a^{2} \mathbf{x}^{2}+C(t), \\
\ddot{a} & =-\frac{4 \pi}{3} G \rho_{0}, \\
H^{2} & =\frac{8 \pi}{3} G \rho_{0}-\frac{K}{a^{2}}
\end{aligned}
$$

where $C(t)$ is an arbitrary function and $K$ is an arbitrary constant. We assume $K=0$ in this paper, corresponding to the Einstein-de Sitter universe. Integrating Eq. (2.7) with Eq. (2.4), we find

$$
a(t)=a_{0} t^{2 / 3}
$$

where $a_{0}$ is a positive constant and the integration constant is chosen so that $a(0)=0$. Equation (2.7), hence, yields

$$
\rho_{0}=\frac{1}{6 \pi G t^{2}}
$$

For the deviation from the zeroth-order solution, we find

$$
\begin{aligned}
& \frac{\partial \mathbf{u}}{\partial t}+H \mathbf{u}+\frac{1}{a}(\mathbf{u} \cdot \nabla) \mathbf{u}=-\frac{1}{a} \nabla \psi, \\
& \frac{\partial \delta}{\partial t}+\frac{1}{a}[\nabla \cdot \mathbf{u}+\nabla \cdot(\delta \mathbf{u})]=0, \\
& \frac{1}{a^{2}} \nabla^{2} \psi=4 \pi G \rho_{0} \delta .
\end{aligned}
$$




\section{B. Linear perturbations}

Linearizing Eqs. (2.10) -(2.12) and denoting linear perturbations with

$$
\mathbf{u}_{1}(t, \mathbf{x})=\sum_{\mathbf{k}} \hat{\mathbf{u}}_{1, \mathbf{k}}(t) e^{i \mathbf{k} \cdot \mathbf{x}}, \delta_{1}(t, \mathbf{x})=\sum_{\mathbf{k}} \hat{\delta}_{1, \mathbf{k}}(t) e^{i \mathbf{k} \cdot \mathbf{x}}, \psi_{1}(t, \mathbf{x})=\sum_{\mathbf{k}} \hat{\psi}_{1, \mathbf{k}}(t) e^{i \mathbf{k} \cdot \mathbf{x}},
$$

we find

$$
\begin{aligned}
& \dot{\hat{\mathbf{u}}}_{1, \mathbf{k}}+H \hat{\mathbf{u}}_{1, \mathbf{k}}=-i \frac{1}{a} \mathbf{k} \hat{\psi}_{1, \mathbf{k}}, \\
& \dot{\hat{\delta}}_{1, \mathbf{k}}+i \frac{1}{a} \mathbf{k} \cdot \hat{\mathbf{u}}_{1, \mathbf{k}}=0, \\
& -\frac{1}{a^{2}} k^{2} \hat{\psi}_{1, \mathbf{k}}=4 \pi G \rho_{0} \hat{\delta}_{1, \mathbf{k}} .
\end{aligned}
$$

Differentiating Eq. (2.15) with respect to $t$ and eliminating $\mathbf{k} \cdot \hat{\mathbf{u}}_{1, \mathbf{k}}$ and $\mathbf{k} \cdot \dot{\hat{\mathbf{u}}}_{1, \mathbf{k}}$ by Eqs. $(2.14)-$ (2.16), we find

$$
\ddot{\hat{\delta}}_{1, \mathbf{k}}+\frac{4}{3 t} \dot{\hat{\delta}}_{1, \mathbf{k}}-\frac{2}{3 t^{2}} \hat{\delta}_{1, \mathbf{k}}=0,
$$

where we have used Eq. (2.8). A general solution is given by

$$
\hat{\delta}_{1, \mathbf{k}}=A_{\mathbf{k}} t^{2 / 3}+B_{\mathbf{k}} t^{-1}
$$

where $A_{\mathbf{k}}$ and $B_{\mathbf{k}}$ are arbitrary constants. The other linear perturbations are given by

$$
\begin{aligned}
& \hat{\psi}_{1, \mathbf{k}}=-\frac{2}{3} \frac{a_{0}^{2}}{k^{2}}\left(A_{\mathbf{k}}+B_{\mathbf{k}} t^{-5 / 3}\right), \\
& \hat{\mathbf{u}}_{1, \mathbf{k}}=i a_{0} \frac{\mathbf{k}}{k^{2}}\left(\frac{2}{3} A_{\mathbf{k}} t^{1 / 3}-B_{\mathbf{k}} t^{-4 / 3}\right)+\mathbf{C}_{\mathbf{k}} t^{-2 / 3},
\end{aligned}
$$

where $\mathbf{C}_{\mathbf{k}}$ is a constant vector satisfying $\mathbf{k} \cdot \mathbf{C}_{\mathbf{k}}=0$. Hereafter, we neglect decaying modes. Then, we find

$$
\mathbf{u}_{1}=-\frac{t}{a} \nabla \psi_{1}
$$

This implies that there is a velocity field potential $\phi=(t / a) \psi_{1}$ such that $\mathbf{u}_{1}=-\nabla \phi$.

\section{ANGULAR MOMENTUM}

Angular momentum within a comoving region $V$ with respect to the origin of the coordinates is given by

$$
\mathbf{L}_{c}=\int_{a^{3} V} \rho \mathbf{r} \times \mathbf{v} d^{3} \mathbf{r}=\rho_{0} a^{4}\left(\int_{V} \mathbf{x} \times \mathbf{u} d^{3} \mathbf{x}+\int_{V} \mathbf{x} \delta \times \mathbf{u} d^{3} \mathbf{x}\right) .
$$

In Sec. III A, we review Peebles's [33] analysis for the second-order contribution to the angular momentum. In Sec. III B, we develop a formulation for the first-order contribution similar to that Catelan and Theuns [34] developed with the Zel'dovich approximation. 


\section{A. Second-order contribution}

If $V$ is a ball centered at the origin, the first term in the parentheses on the rightmost side of Eq. (3.1) vanishes to the first order because of $\mathbf{u}_{1}=-\nabla \phi$. In fact, using Gauss's theorem, we have

$$
\left[\int_{V} \mathbf{x} \times \nabla \phi d^{3} \mathbf{x}\right]_{i}=\int_{\partial V} \epsilon_{i j k} x_{j} \phi d S_{k}
$$

which vanishes if $\partial V$ is a sphere.

To see this term beyond the first order, using Eq. (2.10), we obtain

$$
\frac{d}{d t}\left[a \int_{V} \mathbf{x} \times \mathbf{u} d^{3} \mathbf{x}\right]=-\int_{V} \mathbf{x} \times(\mathbf{u} \cdot \nabla) \mathbf{u} d^{3} \mathbf{x}-\int_{V} \mathbf{x} \times \nabla \psi d^{3} \mathbf{x} .
$$

Then, the second term on the right-hand side of Eq. (3.3) vanishes. To estimate the first term on the right-hand side of Eq. (3.3) to the second order, we can use the solution $\mathbf{u}_{1}$ of the linear perturbation. Since

$$
\left(\mathbf{u}_{1} \cdot \nabla\right) \mathbf{u}_{1}=(\nabla \phi \cdot \nabla) \nabla \phi=\frac{1}{2} \nabla(\nabla \phi)^{2}
$$

the first term on the right-hand side of Eq. (3.3) vanishes to the second order. Thus, we find the contribution from the first term in the parentheses on the rightmost side of Eq. (3.1) is constant to the second order.

The contribution from the second term in the parentheses on the rightmost side of Eq. (3.1) is growing. We should also note that the center of mass is shifted from the origin. The angular momentum $\mathbf{L}$ with respect to the center of mass is then given by

$$
\mathbf{L}=\mathbf{L}_{c}-\mathbf{R} \times \mathbf{P}
$$

where $\mathbf{R}$ is the shift of the center of mass and $\mathbf{P}$ is the linear momentum. We can estimate them to the first order as

$$
\begin{aligned}
& \mathbf{R}=\frac{\int_{a^{3} V} \rho \mathbf{r} d^{3} \mathbf{r}}{\int_{a^{3} V} \rho d^{3} \mathbf{r}}=\frac{a}{V} \int_{V} \mathbf{x} \delta_{1} d^{3} \mathbf{x}, \\
& \mathbf{P}=\int_{a^{3} V} \rho \mathbf{v} d^{3} \mathbf{r}=\rho_{0} a^{3} \int_{V} \mathbf{u}_{1} d^{3} \mathbf{x}+\rho_{0} a^{3} V H \mathbf{R} .
\end{aligned}
$$

To implement the integration, we use the following formula:

$$
\int_{|\mathbf{x}| \leq r_{0}} e^{i \mathbf{k} \cdot \mathbf{x}} d^{3} \mathbf{x}=\frac{4 \pi}{3} r_{0}^{3} g\left(k r_{0}\right) \quad \text { and } \quad \int_{|\mathbf{x}| \leq r_{0}} \mathbf{x} e^{i \mathbf{k} \cdot \mathbf{x}} d^{3} \mathbf{x}=i \frac{4 \pi}{15} r_{0}^{5} f\left(k r_{0}\right) \mathbf{k}
$$


where

$$
g(y):=3\left(\frac{\sin y}{y^{3}}-\frac{\cos y}{y^{2}}\right) \text { and } \quad f(y):=45\left(\frac{\sin y}{y^{5}}-\frac{\cos y}{y^{4}}-\frac{\sin y}{3 y^{3}}\right) .
$$

The functions $f$ and $g$ satisfy

$$
\frac{d g}{d y}=-\frac{y}{5} f, \quad \lim _{y \rightarrow 0} f(y)=\lim _{y \rightarrow 0} g(y)=1, \quad \text { and } \quad \lim _{y \rightarrow \infty} f(y)=\lim _{y \rightarrow \infty} g(y)=0,
$$

and show decaying oscillations. They can be regarded as window functions.

Then, we can show the following result:

$$
\begin{aligned}
\mathbf{R} & =\frac{i}{5} a t^{2 / 3} r_{0}^{2} \sum_{\mathbf{k}} f\left(k r_{0}\right) A_{\mathbf{k}} \mathbf{k}, \\
\mathbf{P} & =\frac{8 \pi}{9} i \rho_{0} a^{4} t^{-1 / 3} r_{0}^{3} \sum_{\mathbf{k}} g\left(k r_{0}\right) A_{\mathbf{k}} \frac{\mathbf{k}}{k^{2}}+\frac{4 \pi}{3} \rho_{0} a^{3} r_{0}^{3} H \mathbf{R}, \\
\mathbf{L}_{c} & =-\frac{8 \pi}{45} \rho_{0}\left(a r_{0}\right)^{5} t^{1 / 3} \sum_{\mathbf{k}, \mathbf{k}^{\prime}} A_{\mathbf{k}} A_{\mathbf{k}^{\prime}} \frac{\mathbf{k} \times \mathbf{k}^{\prime}}{k^{\prime 2}} f\left(\left|\mathbf{k}+\mathbf{k}^{\prime}\right| r_{0}\right) .
\end{aligned}
$$

From Eqs. (3.5) and (3.11)-(3.13), we find

$$
\mathbf{L}=-\frac{8 \pi}{45} \rho_{0}\left(a r_{0}\right)^{5} t^{1 / 3} \sum_{\mathbf{k}, \mathbf{k}^{\prime}} A_{\mathbf{k}} A_{\mathbf{k}^{\prime}} \frac{\mathbf{k} \times \mathbf{k}^{\prime}}{k^{\prime 2}}\left[f\left(\left|\mathbf{k}+\mathbf{k}^{\prime}\right| r_{0}\right)-f\left(k r_{0}\right) g\left(k^{\prime} r_{0}\right)\right]
$$

We can see that $\mathbf{L}$ increases as $t^{5 / 3}$ irrespective of the details of $A_{\mathbf{k}}$. Figure 1 schematically shows that the mode coupling of two independent modes which are not parallel to each other contributes to the growing angular momentum.

Assuming that $A_{\mathbf{k}}$ takes a random phase, we can calculate the variance of $\mathbf{L}$ as follows:

$$
\begin{aligned}
\left\langle\mathbf{L}^{2}\right\rangle & =\left(\frac{8 \pi}{45} \rho_{0}\left(a r_{0}\right)^{5} t^{1 / 3}\right)^{2} \times \sum_{\mathbf{k}_{\mathbf{1}}, \mathbf{k}_{\mathbf{2}}}\left\langle\left|A_{\mathbf{k}_{\mathbf{1}}}\right|^{2}\right\rangle\left\langle\left|A_{\mathbf{k}_{\mathbf{2}}}\right|^{2}\right\rangle\left(\mathbf{k}_{1} \times \mathbf{k}_{2}\right)^{2} \frac{f_{1+2}-f_{1} g_{2}}{k_{2}^{2}} \\
& \times\left[\frac{f_{1+2}-f_{1} g_{2}}{k_{2}^{2}}-\frac{f_{1+2}-f_{2} g_{1}}{k_{1}^{2}}\right],
\end{aligned}
$$

where $f_{1+2}:=f\left(\left|\mathbf{k}_{1}+\mathbf{k}_{2}\right| r_{0}\right), f_{i}:=f\left(k_{i} r_{0}\right)$ and $g_{i}:=f\left(k_{i} r_{0}\right)(i=1,2)$. On the other hand, the density perturbation integrated over the ball of radius $r_{0}$ can be calculated to the first order as

$$
\delta_{s}:=\frac{\int_{a^{3} V} d^{3} \mathbf{r} \rho-\int_{a^{3} V} d^{3} \mathbf{r} \rho_{0}}{\int_{a^{3} V} d^{3} \mathbf{r} \rho_{0}}=\frac{3}{4 \pi r_{0}^{3}} \int_{|\mathbf{x}|<r_{0}} d^{3} \mathbf{x} \delta=t^{2 / 3} \sum_{\mathbf{k}} A_{\mathbf{k}} g\left(k r_{0}\right) .
$$

Thus, the variance of $\delta_{s}$ is given by

$$
\left\langle\delta_{s}^{2}\right\rangle=t^{4 / 3} \sum_{\mathbf{k}}\left\langle\left|A_{\mathbf{k}}\right|^{2}\right\rangle g^{2}\left(k r_{0}\right)
$$




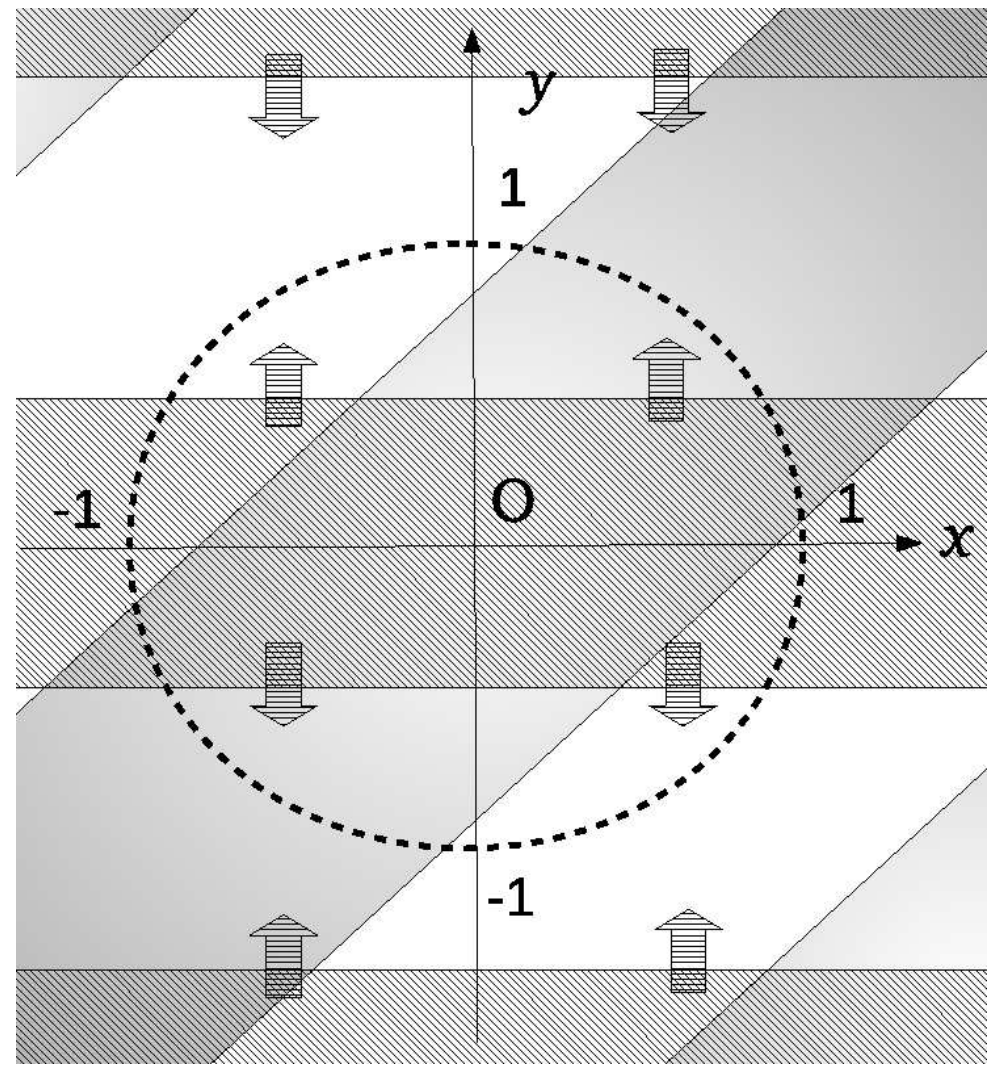

FIG. 1. The second-order contribution to the angular momentum comes from the coupling between two independent modes of linear perturbations. The radius of the ball, $r_{0}$, is chosen to unity. The regions for $\delta>0$ with $\mathbf{k}_{1}=(\pi / 2,-\pi / 2,0)$ are shaded. The regions $\psi>0$ with $\mathbf{k}_{2}=(0, \pi, 0)$ are hatched and the velocity field is denoted by arrows. The density field with $\mathbf{k}_{1}$ and the velocity field with $\mathbf{k}_{2}$ couple with each other so that they compose a growing angular momentum parallel to the $z$ axis in the anticlockwise direction.

From Eqs. (3.15) and (3.17), we find

$$
\left\langle\mathbf{L}^{2}\right\rangle^{1 / 2}=\frac{8 \pi}{45} \rho_{0} \frac{\left(a r_{0}\right)^{5}}{t} \mathcal{I}\left\langle\delta_{s}^{2}\right\rangle,
$$

where we have defined the ratio $\mathcal{I}$ as follows:

$$
\mathcal{I}:=\frac{\left\{\sum_{\mathbf{k}_{1}, \mathbf{k}_{2}}\left\langle\left|A_{\mathbf{k}_{1}}\right|^{2}\right\rangle\left\langle\left|A_{\mathbf{k}_{2}}\right|^{2}\right\rangle\left(\mathbf{k}_{1} \times \mathbf{k}_{2}\right)^{2} \frac{f_{1+2}-f_{1} g_{2}}{k_{2}^{2}}\left[\frac{f_{1+2}-f_{1} g_{2}}{k_{2}^{2}}-\frac{f_{1+2}-f_{2} g_{1}}{k_{1}^{2}}\right]\right\}^{1 / 2}}{\sum_{\mathbf{k}}\left\langle\left|A_{\mathbf{k}}\right|^{2}\right\rangle g^{2}\left(k r_{0}\right)} .
$$

Note that $\mathcal{I}$ does not depend on the overall normalization factor of the power spectrum. We assume that $\mathcal{I}$ is of the order of unity. See [33] for the validity of this assumption. Equation 
(3.18) can be rewritten in the form

$$
\left\langle\mathbf{L}^{2}\right\rangle^{1 / 2}=\frac{2}{15} \mathcal{I} \frac{M R^{2}}{t}\left\langle\delta_{s}^{2}\right\rangle,
$$

where $M:=(4 \pi / 3) \rho_{0}\left(a r_{0}\right)^{3}$ and $R:=a r_{0}$ are the mass and the radius of the ball, respectively. If $\partial V$ is not a sphere, the window functions $f$ and $g$ are modified and the factor $\mathcal{I}$ will be altered. However, even in that case, Eq. (3.20) can still apply as the expression for the second-order contribution.

\section{B. First-order contribution}

If $V$ is not a ball, the first term in the parentheses on the rightmost side of Eq. (3.1) does not vanish in general even in the first order. To estimate this term, we use Eq. (2.21). We also assume that the Maclaurin-series expansion for $\psi_{1}$ is valid over $V$ :

$$
\psi_{1}(\mathbf{x})=\psi_{1}(\mathbf{0})+\partial_{l} \psi_{1}(\mathbf{0}) x_{l}+\frac{1}{2} \partial_{l m}^{2} \psi_{1}(\mathbf{0}) x_{l} x_{m}+O\left(x^{3}\right)
$$

where it should be noted that $\psi_{1}$ is time independent. The truncation of the expansion up to the quadratic terms is justified if the wave number $\mathbf{k}$ of the perturbation satisfies $k r_{0} \lesssim 2 \pi$, where $r_{0}$ here stands for the typical size of the region $V$. For $k r_{0} \gg 2 \pi$, the contribution should cancel out after integration over $V$. Then, we can calculate

$$
-\left[\int_{V} \mathbf{x} \times \nabla \psi_{1} d^{3} \mathbf{x}\right]_{i}=\epsilon_{i j k} D_{j m} J_{k m},
$$

where

$$
J_{j m}:=\int_{V} x_{j} x_{m} d^{3} \mathbf{x} \text { and } \quad D_{k m}:=\partial_{k m}^{2} \psi_{1}(\mathbf{0}) .
$$

To estimate $\mathbf{L}$ to the first order, we assume that the origin is located at the center of mass of $V$. Noting that only the traceless components of $J_{i j}$ and $D_{i j}$ can contribute to $\mathbf{L}$, we finally obtain the first-order term of $\mathbf{L}$ as follows:

$$
\mathbf{L}=t \rho_{0} a^{3} \epsilon_{i j k} \mathcal{D}_{j m} \mathcal{J}_{k m}
$$

where $\mathcal{J}_{i j}:=J_{i j}-\frac{1}{3} \delta_{i j} J_{l l}$ and $\mathcal{D}_{i j}:=D_{i j}-\frac{1}{3} \delta_{i j} D_{l l}$. Note that this contribution grows as $t$.

Assuming that $\partial V$ is determined by an ellipsoid, which is given by

$$
\frac{x_{1}^{2}}{A_{1}^{2}}+\frac{x_{2}^{2}}{A_{2}^{2}}+\frac{x_{3}^{2}}{A_{3}^{2}}=1,
$$


the quadrupole moment $J_{i j}$ of the uniform ellipsoid can be easily calculated to give

$$
\left(J_{i j}\right)=\frac{1}{5} V \operatorname{diag}\left(A_{1}^{2}, A_{2}^{2}, A_{3}^{2}\right)=\operatorname{diag}\left(i_{1}, i_{2}, i_{3}\right),
$$

where the coordinate axes are rotated to the major axes and $V=(4 \pi / 3) A_{1} A_{2} A_{3}$. Assuming that $\mathcal{J}_{i j}$ and $D_{i j}$ are uncorrelated, we obtain

$$
\left\langle\mathbf{L}^{2}\right\rangle=\left(t \rho_{0} a^{3}\right)^{2} \epsilon_{i j k} \epsilon_{i p q}\left\langle D_{j m} D_{p l}\right\rangle \mathcal{J}_{k m} \mathcal{J}_{q l}
$$

We can calculate

$$
\left\langle D_{i j} D_{k l}\right\rangle=\frac{4}{9} a_{0}^{4} \sum_{\mathbf{k}} \frac{k_{i} k_{j} k_{l} k_{m}}{k^{4}}\left\langle\left|A_{\mathbf{k}}\right|^{2}\right\rangle W\left(k r_{0}\right),
$$

where $W\left(k r_{0}\right)$ is a window function which satisfies $W(0)=1$ and falls off for $k r_{0} \rightarrow \infty$. If the power spectrum is isotropic, we can find

$$
\sum_{\mathbf{k}} \frac{k_{i} k_{j} k_{l} k_{m}}{k^{4}}\left\langle\left|A_{\mathbf{k}}\right|^{2}\right\rangle W\left(k r_{0}\right)=\frac{1}{15}\left(\delta_{i j} \delta_{l m}+\delta_{i l} \delta_{j m}+\delta_{i m} \delta_{j l}\right) \sum_{\mathbf{k}}\left\langle\left|A_{\mathbf{k}}\right|^{2}\right\rangle W\left(k r_{0}\right) .
$$

Using the identity

$$
\epsilon_{i j k} \epsilon_{i p q}\left(\delta_{j m} \delta_{p l}+\delta_{j p} \delta_{m l}+\delta_{j l} \delta_{m p}\right) \mathcal{J}_{k m} \mathcal{J}_{q l}=3 \mathcal{J}_{i j} \mathcal{J}_{i j}=2\left(\mu_{1}^{2}-3 \mu_{2}\right),
$$

where $\mu_{1}:=i_{1}+i_{2}+i_{3}$ and $\mu_{2}:=i_{1} i_{2}+i_{2} i_{3}+i_{3} i_{1}$, Eq. (3.27) is transformed to

$$
\left\langle\mathbf{L}^{2}\right\rangle=\left(t \rho_{0} a^{3}\right)^{2} \frac{4}{9} a_{0}^{4} \frac{1}{15} 3 \mathcal{J}_{i j} \mathcal{J}_{i j} \sum_{\mathbf{k}}\left\langle\left|A_{\mathbf{k}}\right|^{2}\right\rangle W\left(k r_{0}\right) .
$$

Thus, we find

$$
\left\langle\mathbf{L}^{2}\right\rangle^{1 / 2} \simeq \sqrt{\frac{2}{15}} \frac{2}{3} a_{0}^{2} t \rho_{0} a^{3}\left(\mu_{1}^{2}-3 \mu_{2}\right)^{1 / 2} \frac{\left\langle\delta_{s}^{2}\right\rangle^{1 / 2}}{t^{2 / 3}},
$$

where we have used Eq. (3.17) with the approximation $W\left(k r_{0}\right) \simeq g^{2}\left(k r_{0}\right)$. We should note $\mu_{1}^{2}-3 \mu_{2} \geq 0$, where the equality holds if and only if $V$ is an exact ball. We can rewrite Eq. (3.32) in the following form:

$$
\left\langle\mathbf{L}^{2}\right\rangle^{1 / 2} \simeq \frac{2}{5 \sqrt{15}} q \frac{M R^{2}}{t}\left\langle\delta_{s}^{2}\right\rangle^{1 / 2},
$$

where we have chosen $r_{0}=\left(A_{1} A_{2} A_{3}\right)^{1 / 3}$ and defined

$$
q:=\sqrt{\frac{\mathcal{J}_{i j} \mathcal{J}_{i j}}{3\left(\frac{1}{5} V r_{0}^{2}\right)^{2}}}=\frac{\sqrt{2\left(\mu_{1}^{2}-3 \mu_{2}\right)}}{\frac{3}{5} V r_{0}^{2}}
$$


as a nondimensional parameter of the initial reduced quadrupole moment of the mass. In Appendix A, we present an exact expression for the first-order contribution for an ellipsoid without invoking the truncated Maclaurin-series expansion and show that Eq. (3.33) is justified if $q$ is not too large.

If we can assume that the center of the volume is located at the peak of the density field and that $\partial V$ is given by an equidensity surface, the distribution of $q$ can be inferred by peak theory [34]. However, we do not need to specify the detailed distribution function of $q$ for the purpose of the current paper.

\section{APPLICATION TO PRIMORDIAL BLACK HOLES}

\section{A. Average angular momentum of masses}

We denote the first-order and second-order contributions, which are given by Eqs. (3.33)

and (3.20), with $\left\langle\mathbf{L}_{(1)}^{2}\right\rangle^{1 / 2}$ and $\left\langle\mathbf{L}_{(2)}^{2}\right\rangle^{1 / 2}$, respectively. It should be noted that $\left\langle\mathbf{L}_{(1)}^{2}\right\rangle^{1 / 2} \propto t$ and $\left\langle\mathbf{L}_{(2)}^{2}\right\rangle^{1 / 2} \propto t^{5 / 3}$. We can understand these two effects in a unified manner. We can see $\left\langle\mathbf{L}_{(1)}^{2}\right\rangle^{1 / 2} \propto a q u_{1} \propto t$, where $q$ is constant in time, while in $\left\langle\mathbf{L}_{(2)}^{2}\right\rangle^{1 / 2}$, the quadrupole moment grows as $t^{2 / 3}$ due to the growth of the density perturbation. This gives time dependence $t \cdot t^{2 / 3}=t^{5 / 3}$ for $\left\langle\mathbf{L}_{(2)}^{2}\right\rangle^{1 / 2}$. Since $\left\langle\mathbf{L}^{2}\right\rangle=\left\langle\mathbf{L}_{(1)}^{2}\right\rangle+\left\langle\mathbf{L}_{(2)}^{2}\right\rangle$, we make an estimate $\left\langle\mathbf{L}^{2}\right\rangle^{1 / 2} \simeq \max \left(\left\langle\mathbf{L}_{(1)}^{2}\right\rangle^{1 / 2},\left\langle\mathbf{L}_{(2)}^{2}\right\rangle^{1 / 2}\right)$.

It would be useful to normalize them at the time of horizon entry $t=t_{H}$, when $R=c H^{-1}$. Then, we find

$$
\begin{aligned}
\left\langle\mathbf{L}_{(1)}^{2}\right\rangle^{1 / 2} & =\frac{2}{5 \sqrt{15}} q \frac{3 G M^{2}}{c} \sigma_{H}\left(\frac{t}{t_{H}}\right) \\
\left\langle\mathbf{L}_{(2)}^{2}\right\rangle^{1 / 2} & =\frac{2}{15} \mathcal{I} \frac{3 G M^{2}}{c} \sigma_{H}^{2}\left(\frac{t}{t_{H}}\right)^{5 / 3}
\end{aligned}
$$

where we have used the relation $\left(a\left(t_{H}\right) r_{0}\right)^{2} / t_{H}=3 G M / c$ and defined $\sigma_{H}:=\left\langle\delta_{s, H}^{2}\right\rangle^{1 / 2}$ with $\delta_{s, H}:=\delta_{s}\left(t_{H}\right)$. Thus, we can estimate the corresponding nondimensional Kerr parameters $a_{*}:=L /\left(G M^{2} / c\right)$ of the mass:

$$
\left\langle a_{*(1)}^{2}\right\rangle^{1 / 2}=\frac{2}{5} \sqrt{\frac{3}{5}} q \sigma_{H}\left(\frac{t}{t_{H}}\right) \text { and }\left\langle a_{*(2)}^{2}\right\rangle^{1 / 2}=\frac{2}{5} \mathcal{I} \sigma_{H}^{2}\left(\frac{t}{t_{H}}\right)^{5 / 3} .
$$

To estimate the final value for the angular momentum, we take the time of maximum expansion $t_{m}$, when nonlinearity becomes important. After this time, we can no longer apply 
linear perturbation theory. The overdense region begins to collapse and separates from the evolution of the rest of the Universe. This implies that the angular momentum becomes almost constant after $t_{m}$.

The average value of $t_{m}$ can be estimated by $\left\langle\delta_{s}^{2}\right\rangle^{1 / 2}=1$ at $t=\left\langle t_{m}\right\rangle$. We find $\left\langle t_{m}\right\rangle=$ $t_{H} \sigma_{H}^{-3 / 2}$ from Eq. (3.17). Thus, we can estimate the average value for the Kerr parameter of the mass as follows:

$$
\left\langle a_{*}^{2}\right\rangle^{1 / 2} \simeq \max \left(\left\langle a_{*(1)}^{2}\right\rangle^{1 / 2},\left\langle a_{*(2)}^{2}\right\rangle^{1 / 2}\right)
$$

where

$$
\left\langle a_{*(1)}^{2}\right\rangle^{1 / 2}=\frac{2}{5} \sqrt{\frac{3}{5}} q \sigma_{H}^{-1 / 2} \text { and }\left\langle a_{*(2)}^{2}\right\rangle^{1 / 2}=\frac{2}{5} \mathcal{I} \sigma_{H}^{-1 / 2} .
$$

If $q=O(1)$, we find that the first-order effect is comparable with the second-order effect. If

we assume $\sigma_{H} \lesssim 0.1$, we have $\left\langle a_{*}^{2}\right\rangle^{1 / 2} \gtrsim 1$, implying that centrifugal force will prevent the direct collapse to a black hole. Only the masses satisfying $a_{*} \leq 1$, which are the minority, can directly collapse to a black hole. Therefore, primordial black hole formation is strongly suppressed by centrifugal force. Most of the primordial black holes are rapidly rotating at least when they are formed. The above argument must be weakened if the matter-dominated era does not last sufficiently long. This possibility will be discussed later.

\section{B. Hypothesis}

Although the above discussion qualitatively indicates the crucial role of angular momentum and the rapid rotation of black holes, it does not tell how the Kerr parameter of the mass is distributed because we have only estimated the average value of $t_{m}$.

To circumvent the difficulty in determining the distributional properties of the Kerr parameter, we make an assumption. In Eqs. (3.14), (3.16), and (3.31), we can see that $\left\langle\mathbf{L}_{(1)}^{2}\right\rangle$, $\left\langle\mathbf{L}_{(2)}^{2}\right\rangle$, and $\left\langle\delta_{s}^{2}\right\rangle$ consist of the coupling of modes. The crucial difference is that $\left\langle\mathbf{L}_{(2)}^{2}\right\rangle$ consists of the mode coupling of two independent modes which are not parallel to each other, while both $\left\langle\mathbf{L}_{(1)}^{2}\right\rangle$ and $\left\langle\delta_{s}^{2}\right\rangle$ consist of the self-coupling of a single mode.

Therefore, despite the complicated dependence of $\mathbf{L}$ on $A_{\mathbf{k}}$, it is natural to assume that $\left|\mathbf{L}_{(1)}\right| \propto \delta_{s}$ and $\left|\mathbf{L}_{(2)}\right| \propto\left\langle\delta_{s}^{2}\right\rangle^{1 / 2} \delta_{s}$, where and hereafter we focus on the overdense regions. More precisely, inspired by Eqs. (3.33) and (3.20), we adopt the following simple approxi- 
mation

$$
\begin{aligned}
& \left|\mathbf{L}_{(1)}\right| \simeq \frac{2}{5 \sqrt{15}} q \frac{M R^{2}}{t} \delta_{s}, \\
& \left|\mathbf{L}_{(2)}\right| \simeq \frac{2}{15} \mathcal{I} \frac{M R^{2}}{t}\left\langle\delta_{s}^{2}\right\rangle^{1 / 2} \delta_{s} .
\end{aligned}
$$

This can be tested by the Monte Carlo simulation. This is consistent with Eqs. (3.33) and (3.20). This also implies that the angular momentum is larger for the mass with larger density perturbation at the same time. Here we explain the motivation of Eq. (4.7). At a density peak, it is most probable that a single mode is excited to a large amplitude, while others are kept to average ones. Note that $\delta_{s}$ can be excited by a single mode, while $\mathbf{L}_{(2)}$ only by the coupling of two independent modes. So, we can estimate $\left|\mathbf{L}_{(2)}\right| \propto\left\langle\delta_{s}^{2}\right\rangle^{1 / 2} \delta_{s}$. This assumption will be justified particularly if $\delta_{s} \gg\left\langle\delta_{s}^{2}\right\rangle^{1 / 2}$. On the other hand, the above approximation is not correct if the wave numbers of all nontrivial modes are parallel to each other, where $\mathbf{L}_{(2)}=0$ but $\delta_{s} \neq 0$. With such an exceptional case, we assume that Eqs. (4.6) and (4.7) are valid for almost all cases.

We estimate $t_{m}=t_{H}\left(\delta_{s, H}\right)^{-3 / 2}$ from Eq. (3.16). Then, from Eqs. (4.6) and (4.7), we can estimate $a_{*}$ as

$$
a_{*} \simeq \max \left(a_{*(1)}, a_{*(2)}\right)
$$

where

$$
a_{*(1)}=\frac{2}{5} \sqrt{\frac{3}{5}} q\left(\delta_{s, H}\right)^{-1 / 2} \text { and } a_{*(2)}=\frac{2}{5} \mathcal{I} \sigma_{H}\left(\delta_{s, H}\right)^{-3 / 2} \text {. }
$$

The dependence on $\delta_{s, H}$ can be understood as follows. Since $t_{m}$ is proportional to $\left(\delta_{s, H}\right)^{-3 / 2}$, it takes a longer time for the mass with smaller $\delta_{s, H}$ to get into the nonlinear regime. This longer $t_{m}$ gives a longer time for the angular momentum to grow and this growth overcompensates the smaller initial value for the seed angular momentum. That is, the smaller $\delta_{s, H}$ is, the larger the final value for $a_{*}$ becomes. This is the case both for the first-order and second-order contributions.

Of course, it should be noted that if $\delta_{s, H}$ is too small, $t_{m}$ can be later than $t_{\text {end }}$, the end time of the matter-dominated era. The finite duration of the matter-dominated era, thus, will give a lower cutoff

$$
\delta_{\mathrm{fd}}:=\left(t_{H} / t_{\mathrm{end}}\right)^{2 / 3}
$$

on $\delta_{s, H}$ below which no primordial black hole is formed. 


\section{Suppression to primordial black hole production}

We can find that the equality $a_{*(1)}=a_{*(2)}$ holds for $\delta_{s, H}=\delta_{s, H t}$, where $\delta_{s, H t}:=$ $\sqrt{5 / 3} \mathcal{I} q^{-1} \sigma_{H}$. This determines a transition point between the two cases $a_{*} \simeq a_{*(1)}>$ $a_{*(2)}$ and $a_{*} \simeq a_{*(2)}>a_{*(1)}$. Thus, if $\delta_{s, H}<\delta_{s, H t}$ for which $a_{* t} \leq a_{*}, a_{*} \simeq a_{*(2)}>$ $a_{*(1)}$, while if $\delta_{s, H}>\delta_{s, H t}$ for which $0 \leq a_{*}<a_{* t}, a_{*} \simeq a_{*(1)}>a_{*(2)}$, where $a_{* t}:=$ $(2 / 5)(3 / 5)^{3 / 4} \mathcal{I}^{-1 / 2} q^{3 / 2} \sigma_{H}^{-1 / 2}$.

We adopt the Kerr bound $a_{*} \leq 1$ as the condition for the direct formation of a black hole. From Eq. (4.9), we can find that this condition reduces to $\delta_{s, H} \geq \delta_{\text {th }}$. The threshold $\delta_{\text {th }}$ is given by

$$
\delta_{\mathrm{th}}=\max \left(\delta_{\mathrm{th}(1)}, \delta_{\mathrm{th}(2)}, \delta_{\mathrm{fd}}\right)
$$

where

$$
\delta_{\operatorname{th}(1)}:=\frac{3 \cdot 2^{2}}{5^{3}} q^{2} \text { and } \delta_{\operatorname{th}(2)}:=\left(\frac{2}{5} \mathcal{I} \sigma_{H}\right)^{2 / 3} .
$$

If $\delta_{\mathrm{fd}}<\delta_{\mathrm{th}(2)}$ or

$$
t_{\text {end }}>\left(\frac{2}{5} \mathcal{I} \sigma_{H}\right)^{-1} t_{H}
$$

we can neglect the effect of finite duration. Otherwise, the primordial black hole formation is significantly suppressed and the tendency towards large spins is weakened. This effect is very sensitive to the cosmological scenario. In Appendix B, we briefly discuss this effect in terms of the reheating temperature. Here we focus on the effect of angular momentum in the following analysis simply by assuming Eq. (4.13). We find that $\delta_{\operatorname{th}(1)}=\delta_{\operatorname{th}(2)}$ if and only if $q=q_{c}$, where

$$
q_{c}=\sqrt{\frac{2}{3}}\left(\frac{5}{2}\right)^{7 / 6} \mathcal{I}^{1 / 3} \sigma_{H}^{1 / 3}
$$

Note that $a_{* t}$ can be rewritten in the form $a_{* t}=\left(q / q_{c}\right)^{3 / 2}$ in terms of $q$ and $q_{c}$. If $q>q_{c}$, $a_{* t}>1$, while if $q<q_{c}, a_{* t}<1$.

Figure 2 schematically shows how the Kerr bound $a_{*} \leq 1$ gives the threshold $\delta_{\text {th }}$ for $\delta_{s, H}$ depending on the value of $q$, where $a_{*} \simeq \max \left(a_{*(1)}, a_{*(2)}\right)$. If $q>q_{c}$, then $\delta_{\operatorname{th}}=\delta_{\operatorname{th}(1)}>\delta_{\operatorname{th}(2)}$, while if $q<q_{c}, \delta_{\mathrm{th}}=\delta_{\operatorname{th}(1)}<\delta_{\operatorname{th}(2)}$.

It is natural to assume that $q_{c}\left(\simeq \sigma_{H}^{1 / 3}\right)$ is small, while there is no a priori reason for $q$ to be perturbatively small. If $q>q_{c}$, we can adopt $\delta_{\mathrm{th}}=\delta_{\mathrm{th}(1)}=\left(3 \cdot 2^{2} / 5^{3}\right) q^{2}$. This implies 


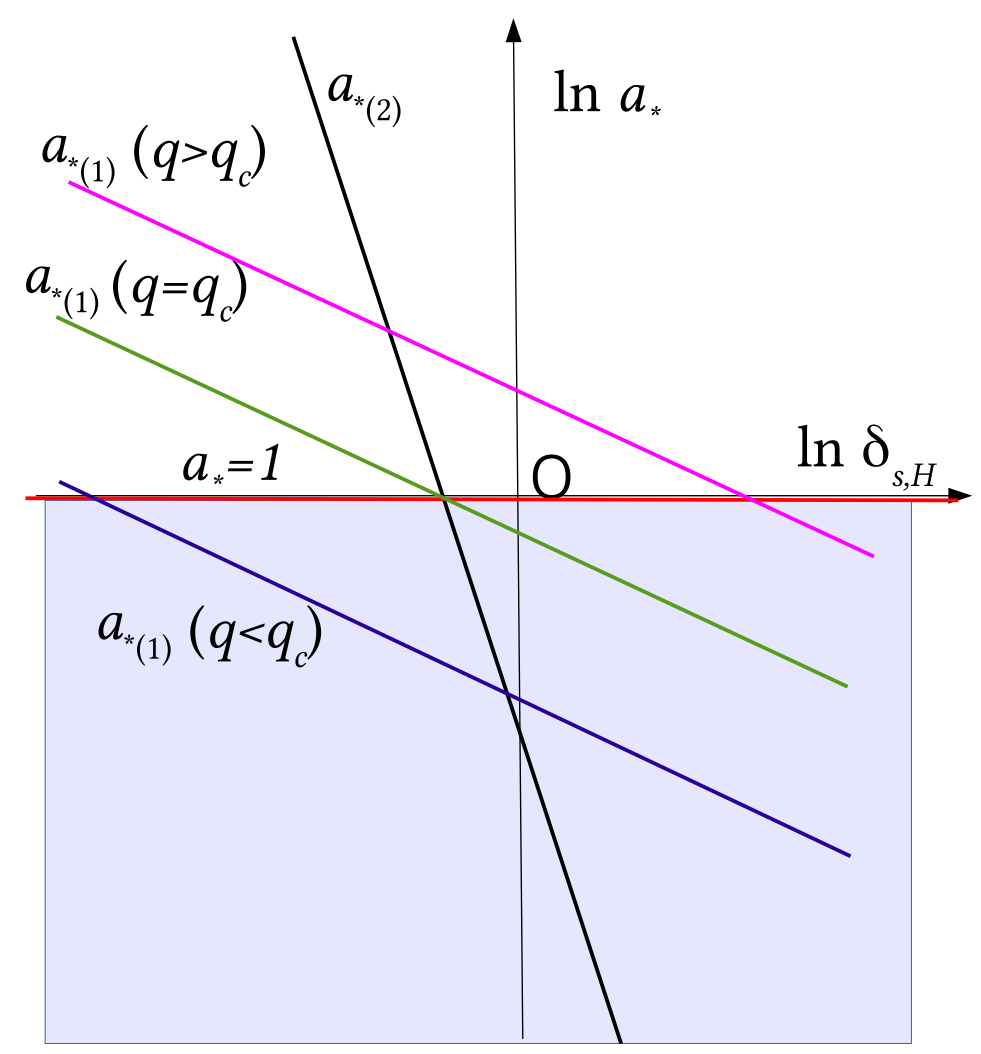

FIG. 2. The first-order and second-order contributions to the Kerr parameter, $a_{*(1)}$ and $a_{*(2)}$, are schematically plotted as functions of the density perturbation at horizon entry, $\delta_{s, H} \cdot a_{*(1)}\left(\delta_{s, H}\right)$ is plotted for three cases, $q>q_{c}, q=q_{c}$, and $q<q_{c}$. The Kerr parameter $a_{*}$ is determined by $a_{*} \simeq \max \left(a_{*(1)}, a_{*(2)}\right)$. The intersection of $a_{*(1)}\left(\delta_{s, H}\right)$ and $a_{*(2)}\left(\delta_{s, H}\right)$ corresponds to the transition point $\left(\delta_{s, H}, a_{*}\right)=\left(\delta_{s, H t}, a_{* t}\right)$. We can see $a_{* t}>1, a_{* t}=1$, and $a_{* t}<1$ for $q>q_{c}, q=q_{c}$, and $q<q_{c}$, respectively. The horizontal line $a_{*}=1$, which is denoted with a red solid line, corresponds to the extreme spin. The region below $a_{*}=1$, which is colored in pale blue, is that for black hole formation satisfying the Kerr bound $a_{*} \leq 1$. We can see that the threshold $\delta_{\text {th }}$ is determined by $a_{*(1)}$ and rapidly increasing with respect to $q$ for $q>q_{c}$, while it is determined by $a_{*(2)}$ and constant for $q \leq q_{c}$.

an exponential suppression factor to black hole formation probability. We use the following formula for a Gaussian distribution:

$$
P=2 \int_{\delta_{\mathrm{th}}}^{\infty} d \delta \frac{1}{\sqrt{2 \pi \sigma^{2}}} \exp \left(-\frac{\delta^{2}}{2 \sigma^{2}}\right)=\operatorname{erfc}\left(\frac{\delta_{\mathrm{th}}}{\sqrt{2} \sigma}\right) \simeq \sqrt{\frac{2}{\pi}} \frac{\sigma}{\delta_{\mathrm{th}}} \exp \left(-\frac{\delta_{\mathrm{th}}^{2}}{2 \sigma^{2}}\right)
$$

where in the last equality we have assumed $\delta_{\text {th }} \gg \sigma$ and used an approximation $\operatorname{erfc}(x) \simeq$ 
$e^{-x^{2}} /(x \sqrt{\pi})$ for $x \gg 1$. This results in the following suppression factor

$$
P_{\mathrm{am}(1)}=\operatorname{erfc}\left(\frac{1}{\sqrt{2} \sigma_{H}} \frac{3 \cdot 2^{2}}{5^{3}} q^{2}\right) \simeq \sqrt{\frac{2}{\pi}}\left(\frac{3 \cdot 2^{2}}{5^{3}}\right)^{-1} q^{-2} \sigma_{H} \exp \left[-\left(\frac{3 \cdot 2^{2}}{5^{3}}\right)^{2} \frac{q^{4}}{2 \sigma_{H}^{2}}\right] .
$$

In the above, we can see that primordial black holes are dominated by masses with smaller $q$. This motivates us to see masses with $q<q_{c}$, which are the minority of all masses.

For $q<q_{c}$, the threshold $\delta_{\text {th }}$ is given by $\delta_{\text {th }}=\delta_{\operatorname{th}(2)}=\left[(2 / 5) \mathcal{I} \sigma_{H}\right]^{2 / 3}$. While this threshold depends on $\sigma_{H}, \delta_{\text {th }} \gg \sigma_{H}$ is still satisfied if $\sigma_{H} \ll 1$. We can estimate a suppression factor through Eq. (4.15) as follows:

$$
P_{\mathrm{am}(2)}=\operatorname{erfc}\left[\frac{1}{\sqrt{2}}\left(\frac{2}{5} \mathcal{I}\right)^{2 / 3} \sigma_{H}^{-1 / 3}\right] \simeq \sqrt{\frac{2}{\pi}}\left(\frac{2}{5} \mathcal{I}\right)^{-2 / 3} \sigma_{H}^{1 / 3} \exp \left[-\frac{1}{2}\left(\frac{2}{5} \mathcal{I}\right)^{4 / 3} \sigma_{H}^{-2 / 3}\right]
$$

Since this suppression is much weaker than that from the first-order effect if $\sigma_{H} \ll 1$, we can conclude that primordial black holes are dominated by the masses with $q<q_{c}$ if $q$ is distributed around 0 , although this suppression factor is still exponential.

\section{Distribution of spins of primordial black holes}

Since

$$
a_{*(1)}^{-2}=\left[\frac{2}{5} \sqrt{\frac{3}{5}} q\right]^{-2} \delta_{s, H} \text { and } a_{*(2)}^{-2 / 3}=\left(\frac{2}{5} \mathcal{I} \sigma_{H}\right)^{-2 / 3} \delta_{s, H},
$$

$a_{*(1)}^{-2}$ and $a_{*(2)}^{-2 / 3}$ obey Gaussian distributions centered at 0 with standard deviations

$$
\sigma_{a_{*(1)}^{-2}}=\left[\frac{2}{5} \sqrt{\frac{3}{5}} q\right]^{-2} \sigma_{H} \text { and } \sigma_{a_{*(2)}^{-2 / 3}}=\left(\frac{2}{5} \mathcal{I}\right)^{-2 / 3} \sigma_{H}^{1 / 3},
$$

respectively, if they are appropriately extended to the whole real axis.

As we have seen, masses with $q<q_{c}$ dominate primordial black holes if $q$ is distributed around 0. In this case, $a_{*} \simeq a_{*(2)} \geq a_{*(1)}$ for $a_{* t} \leq a_{*} \leq 1$, while $a_{*} \simeq a_{*(1)} \geq a_{*(2)}$ for $0 \leq a_{*}<a_{* t}$. For $a_{* t} \leq a_{*} \leq 1$, since $a_{*}^{-2 / 3}$ obeys a Gaussian distribution, we can estimate the probability $f_{\mathrm{BH}}\left(a_{*}\right) d a_{*}$ for $a_{*}$ of the black hole to be between $a_{*}$ and $a_{*}+d a_{*}$ as

$$
f_{\mathrm{BH}(2)}\left(a_{*}\right) d a_{*} \propto \frac{1}{a_{*}^{5 / 3}} \exp \left(-\frac{1}{2 \sigma_{H}^{2 / 3}}\left(\frac{2}{5} \mathcal{I}\right)^{4 / 3} \frac{1}{a_{*}^{4 / 3}}\right) d a_{*} .
$$

For $0 \leq a_{*}<a_{* t}$, since $a_{*}^{-2}$ obeys a Gaussian distribution, we can estimate $f_{\mathrm{BH}}\left(a_{*}\right)$ as

$$
f_{\mathrm{BH}(1)}\left(a_{*}\right) d a_{*} \propto \frac{1}{a_{*}^{3}} \exp \left(-\frac{1}{2 \sigma_{H}^{2}} \frac{3^{2} 2^{4}}{5^{6}} \frac{q^{4}}{a_{*}^{4}}\right) d a_{*} .
$$


Since the distribution function is continuous at $a_{*}=a_{* t}$, we find that $f_{\mathrm{BH}}\left(a_{*}\right)$ is given by

$$
f_{\mathrm{BH}}\left(a_{*}\right)=\left\{\begin{array}{cc}
f_{\mathrm{BH}(1)}\left(a_{*}\right) \frac{f_{\mathrm{BH}(2)}\left(a_{* t}\right)}{f_{\mathrm{BH}(1)}\left(a_{* t}\right)} & \left(0 \leq a_{*}<a_{* t}\right) \\
f_{\mathrm{BH}(2)}\left(a_{*}\right) & \left(a_{* t} \leq a_{*} \leq 1\right)
\end{array}\right.
$$

up to the overall normalization factor. Figure 3 shows the distribution of the black hole spins due to the second-order effect $f_{\mathrm{BH}(2)}\left(a_{*}\right)$, where we have chosen $\mathcal{I}=1$. We can see that most of the black holes are rapidly rotating. For $\sigma_{H}=0.1$, the most frequent value for

the spin is given by $a_{*} \simeq 0.63$. If $\sigma_{H} \gtrsim 0.04$, the most frequent value is smaller than the extreme value $a_{*}=1$, while it becomes the extreme value for $\sigma_{H} \lesssim 0.04$. The distribution becomes sharper and sharper at $a_{*}=1$ as the density fluctuation $\sigma_{H}$ is decreased further. The black hole with $a_{*} \lesssim 0.2$ is very rare for a reasonable range of $\sigma_{H}$. For clarity, we do not plot the switch to the first-order effect for $0 \leq a_{*}<a_{* t}$ in this figure. In fact, the switching for $0 \leq a_{*}<a_{* t}$ does not change the qualitative behavior of the spin distribution function very much. It should be noted that we neglect possible change in the spin due to the general relativistic dynamics of the formation process as well as mass accretion and quantum radiation after formation.

It should be noted that the third-order and higher-order contributions can be as large as the second-order one at the maximum expansion. Generally speaking, higher-order effects will add more variance to the Kerr parameter. This suggests that the current analysis up to the second-order contribution can be valid in order of magnitude, although the higher-order contributions are yet to be studied.

It is also interesting to see black hole spin distribution for $q>q_{c}$, where $a_{*} \simeq a_{*(1)}>a_{*(2)}$ for $0 \leq a_{*} \leq 1$. In this regime, we find $f_{\mathrm{BH}}\left(a_{*}\right)=f_{\mathrm{BH}(1)}\left(a_{*}\right)$ up to the overall normalization. Figure 4 shows the distribution of the black hole spin due to the first-order effect, $f_{\mathrm{BH}(1)}\left(a_{*}\right)$. The exponential dependence indicates that the spin parameter distribution is very dense near $a_{*}=1$, while it is extremely sparse for $a_{*} \lesssim 0.6$. We can see that the tendency towards the extreme rotation is much stronger than that for $f_{\mathrm{BH}(2)}\left(a_{*}\right)$ 


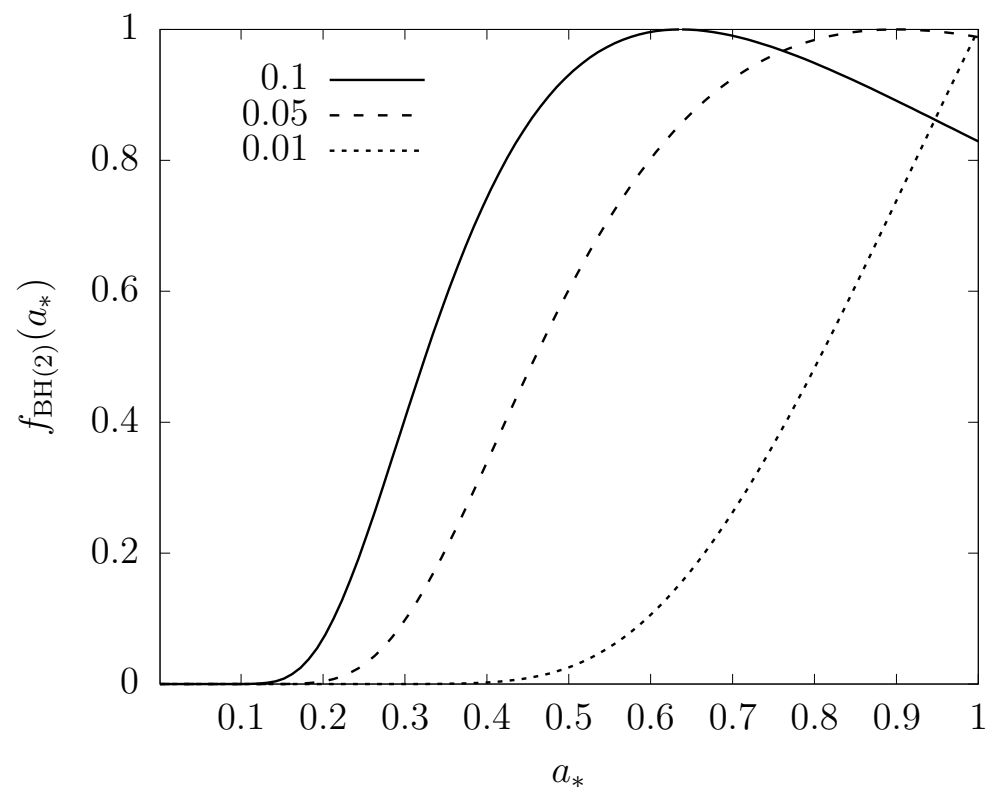

FIG. 3. The spin distribution of primordial black holes formed in the matter-dominated era due to the second-order effect, which applies for $a_{* t}<a_{*}$, where $a_{* t}=\left(q / q_{c}\right)^{3 / 2}$. We put $\mathcal{I}=1$. The curves denote the spin distribution functions normalized by their maximum values for density fluctuations $\sigma_{H}=0.1,0.05$, and 0.01 . We can see that the distribution has a peak at $a_{*} \simeq 0.63$ for $\sigma_{H}=0.1$. The peak value for $a_{*}$ increases as $\sigma_{H}$ is decreased and reaches the extreme value $a_{*}=1$ for $\sigma_{H} \simeq 0.04$. The peak lies at $a_{*}=1$ for $\sigma_{H} \lesssim 0.04$. It becomes sharper and sharper as $\sigma_{H}$ is decreased further. It should be noted that we have neglected possible change in the spin due to the general relativistic dynamics of the formation process as well as mass accretion and quantum radiation after formation.

\section{PRODUCTION RATE}

\section{A. Production rate in the matter-dominated era}

It would be interesting to calculate the probability of black hole formation by combining the effects of angular momentum and anisotropic collapse, the latter of which has been studied by Khlopov and Polnarev [25, 26] and refined by Harada et al. [30].

To proceed further, we briefly introduce the Zel'dovich approximation, where the location of the fluid element is given by

$$
\mathbf{r}=a(t) \mathbf{q}+b(t) \mathbf{p}(\mathbf{q}),
$$




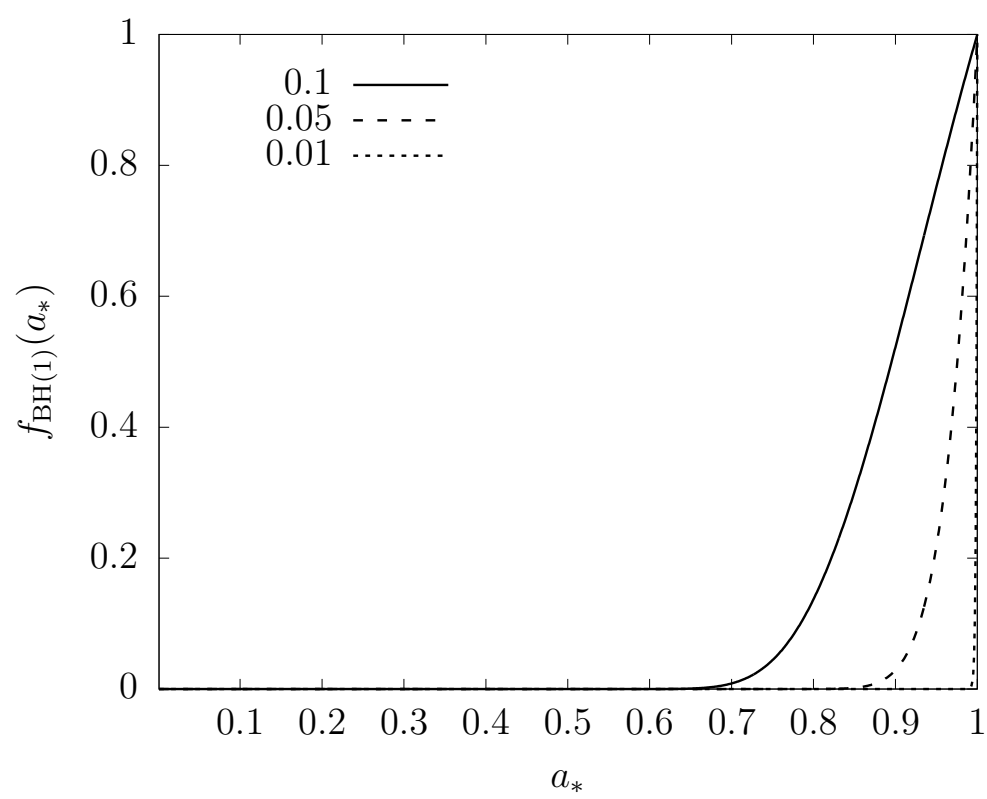

FIG. 4. The spin distribution of primordial black holes formed in the matter-dominated era due to the first-order effect, which applies for $0 \leq a_{*}<a_{* t}$, where $a_{* t}=\left(q / q_{c}\right)^{3 / 2}$. We choose the quadrupole parameter $q$ to $\sqrt{2}$. The curves denote the spin distribution functions normalized by their maximum values for density fluctuations $\sigma_{H}=0.1,0.05$, and 0.01 . We can see that the distribution has a peak at $a_{*}=1$ and it becomes sharper and sharper as $\sigma_{H}$ is decreased. It should be noted that we have neglected possible change in the spin due to the general relativistic dynamics of the formation process as well as mass accretion and quantum radiation after formation.

where $\mathbf{q}$ is the Lagrangian coordinates and $b(t)$ is a growing mode of linear perturbation in Newtonian gravity. We introduce the eigenvalues $\alpha, \beta$, and $\gamma$ of the tensor $-\partial p_{i} / \partial q_{j}$ and assume $\alpha \geq \beta \geq \gamma$ without loss of generality. Taking the normalization $b\left(t_{H}\right)=a\left(t_{H}\right)$, the linear density perturbation at the horizon entry can be given by $\delta_{s, H}(\alpha, \beta, \gamma)=\alpha+\beta+\gamma$. The Zel'dovich approximation [29] is the extrapolation of Eq. (5.1) beyond the linear regime. The probability distribution function for $\alpha, \beta$, and $\gamma$ is given by Doroshkevich [37] as

$$
\begin{aligned}
w(\alpha, \beta, \gamma)= & -\frac{27}{8 \sqrt{5} \pi \sigma_{3}^{6}} \exp \left[-\frac{3}{5 \sigma_{3}^{2}}\left\{\left(\alpha^{2}+\beta^{2}+\gamma^{2}\right)-\frac{1}{2}(\alpha \beta+\beta \gamma+\gamma \alpha)\right\}\right] \\
& \cdot(\alpha-\beta)(\beta-\gamma)(\gamma-\alpha) d \alpha d \beta d \gamma
\end{aligned}
$$

where we can find the relation $\sigma_{H}=\sqrt{5} \sigma_{3}$.

Assuming that a mass to be a black hole is initially given by a ball, the hoop conjecture 
for black hole formation applied to pancake collapse implies $h(\alpha, \beta, \gamma) \lesssim 1$, where

$$
h(\alpha, \beta, \gamma):=\frac{2}{\pi} \frac{\alpha-\gamma}{\alpha^{2}} E\left(\sqrt{1-\left(\frac{\alpha-\beta}{\alpha-\gamma}\right)^{2}}\right)
$$

and $E(e)$ is the complete elliptic integral of the second kind [30]. The production rate $P_{\text {ai }}$ due to this effect is calculated by

$$
P_{\mathrm{ai}} \simeq \int_{0}^{\infty} d \alpha \int_{-\infty}^{\alpha} d \beta \int_{-\infty}^{\beta} d \gamma \theta[1-h(\alpha, \beta, \gamma)] w(\alpha, \beta, \gamma)
$$

Harada et al. numerically calculated this integral and plotted the result in Fig. 1 in [30]. They also obtain a semianalytic formula

$$
P_{\mathrm{ai}} \simeq 0.05556 \sigma_{H}^{5}
$$

In the current paper, we have found that the threshold for black hole formation $\delta_{\text {th }}$ due to the effect of angular momentum is given by Eq. (4.12). Thus, the production rate of primordial black holes can be calculated by

$$
\beta_{0} \simeq \int_{0}^{\infty} d \alpha \int_{-\infty}^{\alpha} d \beta \int_{-\infty}^{\beta} d \gamma \theta\left[\delta_{H}(\alpha, \beta, \gamma)-\delta_{\mathrm{th}}\right] \theta[1-h(\alpha, \beta, \gamma)] w(\alpha, \beta, \gamma)
$$

To see the second-order and first-order effects separately, we put $\delta_{\text {th }}=\delta_{\operatorname{th}(2)}$ and $\delta_{\text {th }}=$ $\delta_{\text {th(1) }}$ in Eq. (5.7) and denote them with $\beta_{0(2)}$ and $\beta_{0(1)}$, respectively. We have numerically implemented triple integration in Eq. (5.4) for $P_{a i}$ and Eq. (5.6) for $\beta_{0(2)}$ and $\beta_{0(1)}$ and plotted the results in Fig. 5 with thick solid lines.

For $\sigma_{H} \ll 1$ and $\delta_{\mathrm{th}} \gg \sigma_{H}$, we have succeeded in deriving the following semianalytic expression for Eq. (5.6):

$$
\beta_{0} \simeq \frac{5 \sqrt{5} \pi^{4}}{(2 \cdot 3)^{9}} \bar{E}^{-5} \frac{\delta_{\mathrm{th}}^{9}}{\sigma_{H}^{4}} \exp \left(-\frac{\delta_{\mathrm{th}}^{2}}{2 \sigma_{H}^{2}}\right)
$$

where $\bar{E} \simeq 1.182$. The derivation of the above formula is described in Appendix C. In Fig. 5, we also plot with dashed lines the semianalytic formula (5.5) for $P_{\text {ai }}$, Eq. (5.7) with $\delta_{\mathrm{th}}=\delta_{\mathrm{th}(2)}$ or

$$
\beta_{0(2)} \simeq 1.921 \times 10^{-7} \mathcal{I}^{6} \sigma_{H}^{2} \exp \left[-0.1474 \frac{\mathcal{I}^{4 / 3}}{\sigma_{H}^{2 / 3}}\right]
$$

for $\beta_{0(2)}$, and Eq. (5.7) with $\delta_{\mathrm{th}}=\delta_{\mathrm{th}(1)}$ or

$$
\beta_{0(1)} \simeq 3.244 \times 10^{-14} \frac{q^{18}}{\sigma_{H}^{4}} \exp \left[-0.004608 \frac{q^{4}}{\sigma_{H}^{2}}\right]
$$


for $\beta_{0(1)}$. For $\beta_{0(2)}$, Eqs. (5.8) and (5.5) agree with the numerical result for $\sigma_{H} \lesssim 0.005$ and for $0.005 \lesssim \sigma_{H} \lesssim 0.2$, respectively. This means that angular momentum is more important for $\sigma_{H} \lesssim 0.005$, while anisotropic collapse is more important for $0.005 \lesssim \sigma_{H} \lesssim 0.2$. Also for $\beta_{0(1)}$, Eqs. (5.9) and (5.5) agree with the numerical result for $\sigma_{H} \lesssim 0.04$ and for $0.04 \lesssim \sigma_{H} \lesssim 0.2$, respectively.

In Fig. 5, we can also see that the suppression due to the second-order effect $\beta_{0(2)}$ is much weaker than that due to the first-order effect $\beta_{0(1)}$ for $\sigma_{H} \lesssim 0.02$. This means that if $\sigma_{H} \lesssim 0.02$ and $q$ is distributed around 0 , the probability of black hole formation is dominated by masses with $q<q_{c} \simeq \sigma_{H}^{1 / 3}$. Therefore, the assumption that the mass is initially given by a ball is naturally justified to estimate the effect of anisotropic collapse. With the distribution of $q$ further taken into account, the probability of black hole formation is semianalytically estimated as

$$
\beta_{0} \simeq\left\{\begin{array}{cc}
1.921 \times 10^{-7} f_{q}\left(q_{c}\right) \mathcal{I}^{6} \sigma_{H}^{2} \exp \left[-0.1474 \frac{\mathcal{I}^{4 / 3}}{\sigma_{H}^{2 / 3}}\right] & \left(\sigma_{H} \lesssim 0.005\right) \\
0.05556 \sigma_{H}^{5} & \left(0.005 \lesssim \sigma_{H} \lesssim 0.2\right) .
\end{array},\right.
$$

where $f_{q}\left(q_{c}\right)$ is the fraction of masses of which $q$ is smaller than $q_{c}$.

For comparison, the production rate in the radiation-dominated phase, $P_{\mathrm{rd}}$, is also plotted with a thin solid line in this figure, where the threshold is chosen to $\delta_{\text {th }}=0.42$. The production rate in the matter-dominated era is larger than that in the radiation-dominated phase for $\sigma_{H} \lesssim 0.05$, while they are comparable with each other for $0.05 \lesssim \sigma_{H} \lesssim 1$.

\section{B. Black hole threshold in the radiation-dominated era}

Here we review black hole threshold in the radiation-dominated phase in terms of density perturbation and curvature perturbation. To define the curvature perturbation, we have to introduce the $3+1$ decomposition of the spacetime

$$
d s^{2}=-\alpha^{2} c^{2} d t^{2}+\psi^{4} a^{2} \tilde{\gamma}_{i j}\left(d x^{i}+\beta^{i} d t\right)\left(d x^{j}+\beta^{j} d t\right),
$$

where we choose $\tilde{\gamma}_{i j}$ so that its determinant equals to that of the flat 3-metric. The curvature perturbation $\zeta$ is defined as

$$
\zeta=-2 \ln \psi
$$




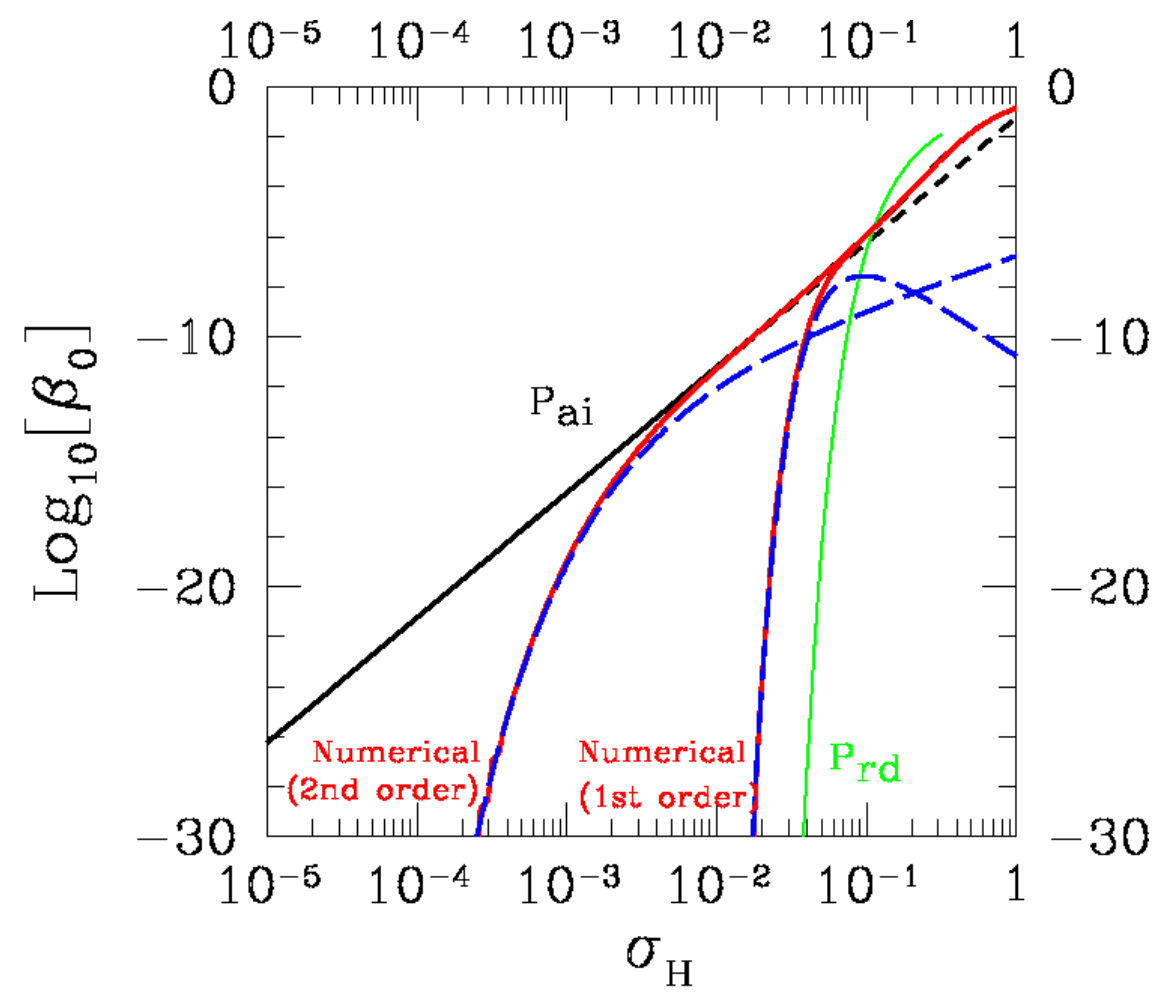

FIG. 5. The production rates of primordial black holes are plotted. We plot the results of numerical integration for $P_{a i}$ due to the effect of anisotropic collapse, $\beta_{0(2)}$ due to the combined effects of anisotropic collapse and second-order angular momentum, and $\beta_{0(1)}$ due to the combined effects of anisotropic collapse and first-order angular momentum with a black solid line labeled $P_{\text {ai }}$, a red solid line labeled "(2nd order)", and a red solid line labeled "(1st order)", respectively. We also plot the corresponding semianalytic formulas with a black short dashed line labeled $P_{\text {ai }}$ and blue long dashed lines labeled "(2nd order)" and "(1st order)", respectively. For comparison, we also plot the production rate in the radiation-dominated era with a green solid line labeled $P_{\mathrm{rd}}$. For $\beta_{0(2)}, \beta_{0(1)}$, and $P_{\mathrm{rd}}$, we choose $\mathcal{I}=1, q=\sqrt{2}$, and $\delta_{\mathrm{th}}=0.42$, respectively. If $q$ is distributed around $0, \beta_{0} \simeq f_{q}\left(q_{c}\right) \beta_{0(2)}$ applies for $\sigma_{H} \lesssim 0.005$, where $f_{q}\left(q_{c}\right)$ denotes the fraction of masses of which $q$ is smaller than $q_{c}$, while $\beta_{0} \simeq P_{\text {ai }}$ applies for $0.005 \lesssim \sigma_{H} \lesssim 1$.

in the uniform-density slicing [38]. Some authors including Kopp et al. [39] take another sign on the right-hand side of Eq. (5.12). Primordial cosmological perturbations are given by long-wavelength solutions, where the length scale of the perturbation is much larger than the Hubble horizon scale [38]. In the lowest and second lowest orders of the long-wavelength limit, $\psi$ is time independent and of the order of unity. The density perturbation $\delta$ in the 
comoving slicing and the $\psi$ are related to each other by the following relation [20]:

$$
\delta=-\frac{4(1+w)}{3 w+5} \frac{c^{2}}{a^{2} H^{2}} \frac{\Delta \psi}{\psi^{5}}
$$

for the equation of state $p=w \rho c^{2}$ with $w$ constant, where $\Delta$ is the Laplacian of the flat 3-metric. This means

$$
\tilde{\delta}:=\lim _{c /\left(a H r_{0}\right) \rightarrow 0}\left(\frac{a H r_{0}}{c}\right)^{2} \delta
$$

is time independent, where $r_{0}$ is the comoving length scale of perturbation and is identified with $r_{0}$ in the previous sections. In general relativistic numerical simulations, $r_{0}$ has been chosen as the radius of the boundary of the overdense region. The threshold for black hole formation has been discussed in terms of $\tilde{\delta}$ after it is averaged within $r_{0}$ and it can be identified with $\delta_{s, H}$ in the previous sections. See $[36,40]$ for the equivalence between the density perturbations in Newtonian gravity and in the comoving slicing in general relativistic cosmological perturbation theory.

By recent numerical relativity simulations in spherical symmetry [20], the black hole threshold in the radiation-dominated era has been found and is $\psi_{\text {th }} \simeq 1.40-1.69$ in terms of the peak value of $\psi$, which is equivalent to $\left|\zeta_{\text {th }}\right| \simeq 0.67-1.05$ in terms of the peak value through Eq. (5.12), depending on the profile of the perturbation. In terms of the density perturbation $\tilde{\delta}$, the threshold is given by $\delta_{\text {th }} \simeq 0.42-0.56$. This result is fairly consistent with preceding works [14-18]. The relation between $\tilde{\delta}$ and the peak value of $\psi$ (or $\zeta$ ) is not one to one but largely profile dependent. In fact, $\delta_{\text {th }}$ and $\psi_{\text {th }}$ even show opposite behaviors on the sharpness of the transition between the overdense region and the flat FriedmannLemaître-Robertson-Walker (FLRW) exterior, as can be seen in Tables I and II and Figs. 2 and 3 in [20]. This suggests that the black hole threshold is profile dependent because of the complexity of gravitational collapse against the pressure gradient force. The analytic formula for a gentle profile is derived using a simple model of perturbation [19] and shows a good agreement with numerical results for $p=w \rho c^{2}$ with $0.01 \leq w \leq 0.6$ obtained by Musco and Miller [18]. This formula gives $\tilde{\delta} \simeq 0.4135$ for the radiation fluid [19].

It should be noted that rather smaller values of $\left|\zeta_{\text {th }}\right|, 0.2131$ for the peak value and 0.0862 for the averaged value, are reported in Sec. IV of [19]. These smaller values are due to the very special conversion function from $\tilde{\delta}$ to $\zeta$ given in [39], which is based on the top-hat curvature profile. In fact, as is shown in [20], this model contains an unphysical feature that the density field has a negative delta-functional term at the transition to the flat FLRW 
exterior and gives a considerably smaller value of $|\zeta|$ for the same $\tilde{\delta}$ than more physical models with smooth and non-negative density fields.

\section{Comparison between the matter-dominated and radiation-dominated eras}

It is useful to discuss the threshold in terms of the Fourier components of the curvature perturbation $\zeta$. Linearizing Eqs. (5.12) and (5.13), we obtain the relation

$$
\hat{\delta}_{\mathbf{k}}=-\frac{2(1+w)}{3 w+5} \frac{c^{2} k^{2}}{(a H)^{2}} \hat{\zeta}_{\mathbf{k}}
$$

where $\hat{\delta}_{\mathbf{k}}$ and $\hat{\zeta}_{\mathbf{k}}$ are the Fourier components of $\delta\left(x^{i}\right)$ and $\zeta\left(x^{i}\right)$, respectively. [A negative sign is missing on the right-hand side of Eq. (56) in [30].] We can express the averaged density perturbation in the real space in terms of the Fourier components of $\zeta$ as

$$
\delta_{s, H}=\lim _{c /\left(a H r_{0}\right) \rightarrow 0}\left(\frac{a H r_{0}}{c}\right)^{2} \sum_{\mathbf{k}} \hat{\delta}_{\mathbf{k}} g\left(k r_{0}\right)=-\frac{2(1+w)}{3 w+5} \sum_{\mathbf{k}}\left(k r_{0}\right)^{2} \hat{\zeta}_{\mathbf{k}} g\left(k r_{0}\right) .
$$

Thus, we find

$$
\sigma_{H}^{2}=\left[\frac{2(1+w)}{3 w+5}\right]^{2} \sum_{\mathbf{k}}\left(k r_{0}\right)^{4}\left\langle\left|\hat{\zeta}_{\mathbf{k}}\right|^{2}\right\rangle g^{2}\left(k r_{0}\right)=\left[\frac{2(1+w)}{3 w+5}\right]^{2} \int_{0}^{\infty} \frac{d k}{k}\left(k r_{0}\right)^{4} P_{\zeta}(k) W\left(k r_{0}\right),
$$

where $W\left(k r_{0}\right)$ is identified with $g^{2}\left(k r_{0}\right)$. We have assumed a random phase and isotropy in the distribution of $\hat{\zeta}_{\mathbf{k}}$ and defined the power spectrum $P_{\zeta}(k):=\left[k^{3} /\left(2 \pi^{2}\right)\right]\left\langle\left|\hat{\zeta}_{k}\right|^{2}\right\rangle$. Because of the rapidly increasing function $k^{4}$ and the window function in Eq. (5.17), the right-hand side can be written by the power spectrum at the characteristic wave number $k=k_{\mathrm{BH}}$. Although there appears subtlety in identifying $k_{\mathrm{BH}}$, we simply write Eq. (5.17) as

$$
\sigma_{H}^{2} \simeq\left[\frac{2(1+w)}{3 w+5}\right]^{2} P_{\zeta}\left(k_{\mathrm{BH}}\right)
$$

Note that this agrees with Eq. (3.5) of Alabidi et al. [24] up to a factor of 2, which will depend on the definition of $k_{\mathrm{BH}}$. This $\sigma_{H}$ can be directly compared with the threshold $\delta_{\mathrm{th}}$.

Based on the above argument, let us compare the production rates for the two eras. In the radiation-dominated era, we find $\sigma_{H}^{2} \simeq(16 / 81) P_{\zeta}$. From Eq. (5.16), we can have an approximate relation

$$
\delta_{s, H} \simeq-\left.\frac{2(1+w)}{3 w+5} \hat{\zeta}_{\mathbf{k}}\right|_{k=k_{\mathrm{BH}} \simeq r_{0}^{-1}}
$$


which is consistent with Eq. (5.18). Therefore, the black hole threshold $\delta_{\text {th }} \simeq 0.42-0.56$ in the radiation-dominated era is roughly equivalent to $\left|\hat{\zeta}_{\mathbf{k}}\right|_{\text {th }} \simeq 0.95-1.26$ by Eq. (5.16). In the matter-dominated era, we have $\sigma_{H}^{2}=(4 / 25) P_{\zeta}$. In the current paper, we find that for $q<q_{c}$, the threshold is given by $\delta_{\text {th }}=\left(2 \mathcal{I} \sigma_{H} / 5\right)^{2 / 3}$, which is roughly equivalent to $\left|\hat{\zeta}_{\mathbf{k}}\right|_{\text {th }} \simeq$ $0.74 \mathcal{I}^{2 / 3} P_{\zeta}^{1 / 3}$ through Eqs. (5.18) and (5.19), while for $q>q_{c}$, it is given by $\delta_{\mathrm{th}} \simeq 0.096 q^{2}$, which is roughly equivalent to $\left|\hat{\zeta}_{\mathbf{k}}\right|_{\text {th }} \simeq 0.24 q^{2}$. If $q$ is distributed around 0 , we can conclude that black hole production is enhanced in the matter-dominated phase in comparison with the radiation-dominated phase because some fraction of masses have $q<q_{c}$ and those masses dominate the probability of black hole formation and give a larger production rate than in the radiation-dominated era. Even if $q<q_{c}$ is highly restricted, the masses with $q$ satisfying $q_{c}<q \lesssim 2.0-2.2$, which have the threshold value $\left|\hat{\zeta}_{\mathbf{k}}\right|_{\text {th }}$ smaller than that in the radiation-dominated era, will dominate the production rate and give a larger production rate.

\section{CONCLUSION}

We conclude that angular momentum plays crucial roles in primordial black hole formation in the matter-dominated phase of the Universe if it lasts sufficiently long. In fact, the formation of primordial black holes is exponentially suppressed contrary to conventional expectations. This suppression is much stronger than the effect of anisotropic collapse and the conventional formula overestimates the production rate. However, since the newly obtained exponential suppression is much weaker than that in the radiation-dominated era, the matter-dominated era can still be regarded as the epoch of enhanced production of primordial black holes. We also find that most of the primordial black holes formed in the matter-dominated era were rapidly rotating at their formation epoch and still are if they have kept a large fraction of spins until now. This has interesting implications for astrophysics and cosmology. We also predict that when primordial black holes are formed, much more "minihaloes" are formed, which have supercritical values of the Kerr parameter. If the matter-dominated era does not last so long, the production rate of primordial black holes is strongly suppressed and the tendency towards large spins of both the primordial black holes and the minihaloes is significantly weakened. Since the duration of the matter-dominated era is highly dependent on the cosmological scenario, it would be very interesting from a 
cosmological point of view to investigate the finite duration effect on the spins of primordial black holes.

\section{ACKNOWLEDGMENTS}

The authors are grateful to C. Byrnes, B. J. Carr, J. Garriga, T. Hiramatsu, T. Igata, S. Jhingan, T. Kobayashi, I. Musco, T. Nakama, M. Sasaki, T. Suyama, T. Tanaka, and S. Yokoyama for helpful comments. The authors also thank Tommi Tenkanen and Takahiro Terada. This work was supported by JSPS KAKENHI Grants No. JP26400282 (T.H.), No. JP16K17688, No. JP16H01097 (C.Y.), No. JP26247041, No. JP15H05889, No. JP16H0877, No. JP17H01131 (K.K.), and No. JP25400265 (K.N.).

\section{Appendix A: Exact expression for the first-order contribution in an ellipsoid}

First, we derive exact expressions for the integrals given in Eq. (3.8), where the region of integration is that inside the ellipsoid given by Eq. (3.25). We replace the integration variable $\mathbf{x}$ by $\mathbf{y}$ defined as $\mathbf{x}=:\left(A_{1} y_{1}, A_{2} y_{2}, A_{3} y_{3}\right)$ and introduce a new wave number defined as $\tilde{\mathbf{k}}:=\left(A_{1} k_{1}, A_{2} k_{2}, A_{3} k_{3}\right)$. Then, we have

$$
\begin{aligned}
& \int_{V} e^{i \mathbf{k} \cdot \mathbf{x}} d^{3} \mathbf{x}=A_{1} A_{2} A_{3} \int_{|\mathbf{y}| \leq 1} e^{-\tilde{\mathbf{k}} \cdot \mathbf{y}} d^{3} \mathbf{y}=V g(\tilde{k}), \\
& \int_{V} x_{i} e^{i \mathbf{k} \cdot \mathbf{x}} d^{3} \mathbf{x}=A_{1} A_{2} A_{3} A_{i} \int_{|\mathbf{y}| \leq 1} y_{i} e^{-\tilde{\mathbf{k}} \cdot \mathbf{y}} d^{3} \mathbf{y}=\frac{i}{5} V \bar{k}_{i} f(\tilde{k}),
\end{aligned}
$$

where $\tilde{k}:=\sqrt{\left(A_{1} k_{1}\right)^{2}+\left(A_{2} k_{2}\right)^{2}+\left(A_{3} k_{3}\right)^{2}}$ and $\overline{\mathbf{k}}:=\left(A_{1}^{2} k_{1}, A_{2}^{2} k_{2}, A_{3}^{2} k_{3}\right)$. Hence, the averaged density perturbation and its variance are given by

$$
\delta_{s}=\sum_{\mathbf{k}} A_{\mathbf{k}} g(\tilde{k}) \quad \text { and } \quad\left\langle\delta_{s}^{2}\right\rangle=\sum_{\mathbf{k}}\left\langle\left|A_{\mathbf{k}}\right|^{2}\right\rangle g^{2}(\tilde{k})
$$

respectively, where $A_{\mathbf{k}}$ is assumed to take a random phase. We also have

$$
\int_{V} \epsilon_{i j l} x_{j} \partial_{j} \psi_{l} d^{3} \mathbf{x}=\epsilon_{i j l} \sum_{\mathbf{k}} i k_{l} \hat{\psi}_{\mathbf{k}} \int_{V} x_{j} e^{i \mathbf{k} \cdot \mathbf{x}} d^{3} \mathbf{x}=\frac{2}{15} V a_{0}^{2} \epsilon_{i j l} \sum_{\mathbf{k}} \frac{\bar{k}_{j} k_{l}}{k^{2}} f(\tilde{k}) A_{\mathbf{k}} .
$$

The first-order contribution to the angular momentum $\mathbf{L}_{(1)}$ is then given by

$$
L_{(1) i}=-\frac{2}{15} V \rho_{0} a^{3} t a_{0}^{2} \epsilon_{i j l} \sum_{\mathbf{k}} \frac{\bar{k}_{j} k_{l}}{k^{2}} f(\tilde{k}) A_{\mathbf{k}}
$$


From Eq. (A5), we have its variance

$$
\begin{aligned}
\left\langle\mathbf{L}_{(1)}^{2}\right\rangle & =\left(\frac{2}{3} \rho_{0} a^{3} a_{0}^{2} t\right)^{2} \sum_{\mathbf{k}} \frac{f^{2}(\tilde{k})}{k^{4}}\left\langle\left|A_{\mathbf{k}}\right|^{2}\right\rangle \\
& \times\left[\left(i_{1}-i_{2}\right)^{2}\left(k_{1} k_{2}\right)^{2}+\left(i_{2}-i_{3}\right)^{2}\left(k_{2} k_{3}\right)^{2}+\left(i_{3}-i_{1}\right)^{2}\left(k_{3} k_{1}\right)^{2}\right] \\
& =\left(t \rho_{0} a^{3}\right)^{2} \frac{4}{9} a_{0}^{4} \epsilon_{i j k} \epsilon_{i p q} J_{k m} J_{q l} \sum_{\mathbf{k}} f^{2}(\tilde{k}) \frac{k_{j} k_{m} k_{p} k_{l}}{k^{4}}\left\langle\left|A_{\mathbf{k}}\right|^{2}\right\rangle .
\end{aligned}
$$

If the eccentricity is low, or equivalently $q$ is small, we can neglect the anisotropy in $f^{2}(\tilde{k})$ and we have

$$
\left\langle\mathbf{L}_{(1)}^{2}\right\rangle=\frac{2}{15}\left(\frac{2}{3} \rho_{0} a^{3} a_{0}^{2} t\right)^{2}\left(\mu_{1}^{2}-3 \mu_{2}\right) \sum_{\mathbf{k}}\left\langle\left|A_{\mathbf{k}}\right|^{2}\right\rangle f^{2}\left(k r_{0}\right),
$$

where the power spectrum is assumed to be isotropic. However, if the eccentricity is high, or equivalently, $q$ is large, we cannot neglect the anisotropy in $f^{2}(\tilde{k})$. Even in this case, however, we may still rewrite Eq. (A6) in the following form:

$$
\left\langle\mathbf{L}_{(1)}^{2}\right\rangle^{1 / 2}=\frac{2}{5 \sqrt{15}} \mathcal{R} q \frac{M R^{2}}{t}\left\langle\delta_{\mathrm{s}}^{2}\right\rangle^{1 / 2}
$$

where

$$
\mathcal{R}:=\sqrt{\frac{\epsilon_{i j k} \epsilon_{i p q} J_{k m} J_{q l} \sum_{\mathbf{k}} f^{2}(\tilde{k}) \frac{k_{j} k_{m} k_{p} k_{l}}{k^{4}}\left\langle\left|A_{\mathbf{k}}\right|^{2}\right\rangle}{\epsilon_{i j k} \epsilon_{i p q} J_{k m} J_{q l} \sum_{\mathbf{k}} f^{2}\left(k r_{0}\right) \frac{k_{j} k_{m} k_{p} k_{l}}{k^{4}}\left\langle\left|A_{\mathbf{k}}\right|^{2}\right\rangle} \frac{\sum_{\mathbf{k}} f^{2}\left(k r_{0}\right)\left\langle\left|A_{\mathbf{k}}\right|^{2}\right\rangle}{\sum_{\mathbf{k}} g^{2}(\tilde{k})\left\langle\left|A_{\mathbf{k}}\right|^{2}\right\rangle}} .
$$

$\mathcal{R}$ does not depend on the overall normalization factor. If $\mathcal{R} \simeq 1$, we recover Eq. (3.33).

\section{Appendix B: Condition for the end time in terms of the reheating temperature}

As an interesting example, in this section we assume that the reheating process due to a decay of massive particles makes the radiation-dominated phase start, i.e., $t_{\text {end }}=t_{R}$, where $t_{R}$ is the cosmic time at the reheating.

In this case, for the wave number $k$ crossing the horizon $(k=a H)$ at $t=t_{H}$ during the matter-dominated phase, the relation between $k$ and $t_{H}$ is given by

$$
k \sim k_{\mathrm{eq}}\left(\frac{T_{R}}{T_{\mathrm{eq}}}\right)\left(\frac{t_{H}}{t_{R}}\right)^{-1 / 3},
$$

where $k_{\text {eq }}$ and $T_{\text {eq }}$ are the wave number and the temperature at the (latest) matter-radiation equality, respectively. Then, we can show that Eq. (4.13) gives an upper bound on the reheating temperature $T_{R}$ for the successful enhanced production of highly spinning primordial 
black holes as

$$
T_{R} \lesssim 0.1 \mathrm{GeV}\left(\frac{k}{10^{6} \mathrm{Mpc}^{-1}}\right)\left(\frac{2}{5} \mathcal{I} \sigma_{H}\right)^{1 / 3}
$$

where we have used the relation between $t_{R}$ and $T_{R}$

$$
t_{R} \simeq\left(\frac{g_{*}}{45 /\left(2 \pi^{2}\right)}\right)^{-1 / 2} \frac{m_{\mathrm{Pl}}}{T_{R}^{2}} \sim \frac{m_{\mathrm{Pl}}}{T_{R}^{2}}
$$

with $g_{*} \simeq 10.75-106.75$ and put $m_{\mathrm{Pl}} \simeq 2.4 \times 10^{18} \mathrm{GeV}, k_{\mathrm{eq}} \sim 0.01 \mathrm{Mpc}^{-1}$, and $T_{\mathrm{eq}} \sim 0.7 \mathrm{eV}$.

\section{Appendix C: Derivation of the semianalytic formula}

Changing the variables from $(\alpha, \beta, \gamma)$ to $(x, y, z)$ by

$$
x=\frac{\alpha+\beta+\gamma}{3}, \quad y=\frac{(\alpha-\beta)-(\beta-\gamma)}{4}, \quad z=\frac{\alpha-\gamma}{2}
$$

and from $(x, y, z)$ to $(t, u, z)$ by

$$
t=\frac{x}{z}, \quad u=\frac{y}{z},
$$

we find that the distribution function for $(t, u, z)$ is given by

$$
\tilde{w}(t, u, z) d t d u d z=-\frac{27}{\sqrt{5} \pi \sigma_{3}^{6}}(2 u-1)(2 u+1) z^{5} \exp \left[-A(t, u) z^{2}\right] d t d u d z
$$

where

$$
A(t, u):=\frac{9}{10}\left(\frac{t}{\sigma_{3}}\right)^{2}+2\left(\frac{u}{\sigma_{3}}\right)^{2}+\frac{3}{2}\left(\frac{1}{\sigma_{3}}\right)^{2}
$$

and the domain $\infty>\alpha \geq \beta \geq \gamma \geq-\infty$ is transformed to $-\infty<x<\infty,-1 / 2<u<1 / 2$, and $0<z<\infty$. Since

$$
h(\alpha, \beta, \gamma)=\tilde{h}(t, u, z):=\frac{4}{\pi z}\left(t+\frac{2}{3} u+1\right)^{-2} E\left(\sqrt{1-\left(u+\frac{1}{2}\right)^{2}}\right),
$$

the criterion for the black hole formation, $h<1$, is transformed to

$$
z>z_{*}(t, u):=\frac{4}{\pi}\left(t+\frac{2}{3} u+1\right)^{-2} \tilde{E}(u),
$$

where we put

$$
\tilde{E}(u)=E\left(\sqrt{1-\left(u+\frac{1}{2}\right)^{2}}\right) .
$$


If we take only the anisotropic collapse into account, we find

$$
\beta_{0}=-\frac{27}{\sqrt{5} \pi \sigma_{3}^{6}} \int_{-1 / 2}^{1 / 2} d u(2 u-1)(2 u+1) \int_{-1-(2 / 3) u}^{\infty} d t \int_{z_{*}(t, u)}^{\infty} d z z^{5} \exp \left[-A(t, u) z^{2}\right] .
$$

For $\sigma_{H}=\sqrt{5} \sigma_{3} \ll 1$, we can obtain the semianalytic formula

$$
\beta_{0} \simeq \frac{5 \cdot 5^{3} \pi^{9 / 2}}{2^{9} \cdot 3^{6} \sqrt{10}} \bar{E}^{-5} \sigma_{H}^{5} \simeq 0.05556 \sigma^{5}
$$

where

$$
\bar{E}^{-5}:=\frac{3}{2} \int_{-1 / 2}^{1 / 2}(1-2 u)(1+2 u) \tilde{E}^{-5}(u)
$$

and $\bar{E} \simeq 1.182$. The derivation of the above formula is given in Appendix B of [30].

To take both anisotropic collapse and angular momentum into account, we would like to calculate the integral in Eq. (5.6). This can be rewritten as

$$
\beta_{0}=-\frac{27}{\sqrt{5} \pi \sigma_{3}^{6}} \int_{-1 / 2}^{1 / 2} d u(2 u-1)(2 u+1) \int_{-1-(2 / 3) u}^{\infty} d t \int_{z_{0}(t, u)}^{\infty} d z z^{5} \exp \left[-A(t, u) z^{2}\right]
$$

where $z_{0}(t, u):=\max \left(z_{*}(t, u), z_{\mathrm{th}}(t)\right)$ and $z_{\mathrm{th}}(t):=\delta_{\mathrm{th}} /(3 t)$. We denote two roots of $z_{*}(t, u)=z_{\text {th }}(t)$ with $t_{1}(u)$ and $t_{2}(u)\left(t_{1}(u)<t_{2}(u)\right)$. We can find

$$
t_{1,2}(u)=\frac{18 \tilde{E}(u)-(3+2 u) \pi \delta_{\mathrm{th}} \mp 6 \sqrt{\tilde{E}(u)\left(9 \tilde{E}(u)-(3+2 u) \pi \delta_{\mathrm{th}}\right)}}{3 \delta_{\mathrm{th}}},
$$

where $t_{1}(u)$ and $t_{2}(u)$ correspond to the upper and lower signs, respectively. We have $z_{0}(t, u)=z_{*}(t, u)$ for $-1-(2 / 3) u<t<0$ and $t_{1}(u)<t<t_{2}(u)$, while $z_{0}(t, u)=z_{\text {th }}(t)$ for $0<t<t_{1}(u)$ and $t_{2}(u)<t$. The integration with respect to $z$ can be done explicitly using a well-known formula, which is given by Eq. (66) in Appendix A of [30]. The result is

$$
\begin{aligned}
\beta_{0}= & -\frac{27}{2 \sqrt{5} \pi \sigma_{3}^{6}} \int_{-1 / 2}^{1 / 2} d u(2 u-1)(2 u+1) \\
& \times\left[\left(\int_{-1-(2 / 3) u}^{\infty}+\int_{t_{1}(u)}^{t_{2}(u)}\right) d t F\left(A, z_{*}\right)+\left(\int_{0}^{t_{1}(u)}+\int_{t_{2}(u)}^{\infty}\right) F\left(A, z_{\mathrm{th}}\right)\right],
\end{aligned}
$$

where

$$
F(A, z):=\frac{2+2 A z^{2}+A^{2} z^{4}}{A^{3}} \exp \left[-A z^{2}\right]
$$

The above expression is a result of exact transformation from Eq. (5.6).

Hereafter, we assume $\sigma_{3} \ll 1$ and $\delta_{\mathrm{th}} \gg \sigma_{3}$ and look for an approximate expression for $\beta_{0}$. From the behavior of $A(t, u) z_{0}^{2}(t, u)$, we can show that the dominant contribution can 
come from the integral of the interval $\left[t_{1}(u), t_{2}(u)\right]$ or $\left[t_{2}(u), \infty\right)$ on the right-hand side of Eq. (C13). We denote the former and latter contributions to $\beta_{0}$ with $I_{1}$ and $I_{2}$, respectively. For $t \gtrsim 1$, we obtain

$$
A \simeq \frac{9 t^{2}}{10 \sigma_{3}^{2}}, \quad z_{*} \simeq \frac{4}{\pi t^{2}} \tilde{E}(u)
$$

and, hence,

$$
A z_{*}^{2} \simeq \frac{72 \tilde{E}(u)}{\pi^{2} \sigma^{2} t^{2}}, \quad A z_{\mathrm{th}}^{2} \simeq \frac{\delta_{\mathrm{th}}^{2}}{10 \sigma_{3}^{2}}, \quad t_{2}(u) \simeq \frac{12 \tilde{E}(u)}{\pi \delta_{\mathrm{th}}},
$$

where we have used $z_{*}\left(t_{2}(u), u\right)=z_{\mathrm{th}}\left(t_{2}(u)\right)$. Then, $I_{2}$ is calculated to give

$$
I_{2} \simeq \frac{5 \sqrt{5} \pi^{4}}{(2 \cdot 3)^{9}} \bar{E}^{-5} \frac{\delta_{\mathrm{th}}^{9}}{\sigma_{H}^{4}} \exp \left(-\frac{\delta_{\mathrm{th}}^{2}}{2 \sigma_{H}^{2}}\right),
$$

where we have used $\delta_{\mathrm{th}} \gg \sigma_{H}$. On the other hand, we can estimate $I_{1}$ as

$$
I_{1} \sim \frac{\delta_{\mathrm{th}}^{7}}{\sigma_{H}^{2}} \exp \left(-\frac{\delta_{\mathrm{th}}^{2}}{2 \sigma_{H}^{2}}\right)
$$

up to a numerical factor of the order of unity and this is clearly subdominant to $I_{2}$. Thus, we finally reach Eq. (5.7). The discussion does not change whether $\delta_{\mathrm{th}}=O(1)$ or $\delta_{\mathrm{th}}=O\left(\sigma_{H}^{2 / 3}\right)$.

[1] B. J. Carr, K. Kohri, Y. Sendouda and J. Yokoyama, Phys. Rev. D 81104019 (2010)

[2] B. J. Carr, F. Kühnel and M. Sandstad, Phys. Rev. D 94 (2016) no.8, 083504

[3] B. Carr, M. Raidal, T. Tenkanen, V. Vaskonen and H. Veermäe, Phys. Rev. D 96 (2017) no.2, 023514

[4] M. Sasaki, T. Suyama, T. Tanaka, and S. Yokoyama, Phys. Rev. Lett. 117061101 (2016) arXiv:1603.08338 [astro-ph.CO].

[5] B. P. Abbott et al. [LIGO Scientific and Virgo Collaborations], Phys. Rev. Lett. 116 (2016) no.6, 061102

[6] S. Bird, I. Cholis, J. B. Muñoz, Y. Ali-Haïmoud, M. Kamionkowski, E. D. Kovetz, A. Raccanelli and A. G. Riess, Phys. Rev. Lett. 116, no. 20, 201301 (2016)

[7] S. Clesse and J. García-Bellido, Phys. Dark Univ. 15, 142 (2017)

[8] M. Raidal, V. Vaskonen and H. Veermäe, JCAP 1709, 037 (2017)

[9] B. P. Abbott et al. [LIGO Scientific and VIRGO Collaborations], Phys. Rev. Lett. 118 (2017) no.22, 221101

[10] P. Pani and A. Loeb, Phys. Rev. D 88 (2013) 041301 
[11] T. Chiba and S. Yokoyama, Prog. Theor. Exp. Phys. 2017, $083 E 01$ (2017).

[12] C. Gundlach and T. W. Baumgarte, Phys. Rev. D 94 (2016) no.8, 084012

[13] B. J. Carr, Astrophys. J. 2011 (1975)

[14] M. Shibata and M. Sasaki, Phys. Rev. D 60084002 (1999)

[15] I. Musco, J. C. Miller, and L. Rezzolla, Class. Quant. Grav. 221405 (2005)

[16] A. G. Polnarev and I. Musco, Class. Quant. Grav. 241405 (2007)

[17] I. Musco, J. C. Miller, and A. G. Polnarev, Class. Quant. Grav. 26235001 (2009)

[18] I. Musco and J. C. Miller, Class. Quant. Grav. 30145009 (2013)

[19] T. Harada, C.-M. Yoo and K. Kohri, Phys. Rev. D 88084051 (2013) Erratum: [Phys. Rev. D 89 029903]

[20] T. Harada, C.-M. Yoo, T. Nakama and Y. Koga, Phys. Rev. D 91084057 (2015)

[21] H. Assadullahi and D. Wands, Phys. Rev. D 81, 023527 (2010)

[22] L. Alabidi and K. Kohri, Phys. Rev. D 80063511 (2009)

[23] L. Alabidi, K. Kohri, M. Sasaki, and Y. Sendouda, JCAP 1209017 (2012)

[24] L. Alabidi, K. Kohri, M. Sasaki and Y. Sendouda, JCAP 1305033 (2013)

[25] M. Y. Khlopov and A. G. Polnarev, Phys. Lett. B 97383 (1980)

[26] A. G. Polnarev and M. Y. Khlopov, Sov. Astron. 269 (1982)

[27] J. L. G. Sobrinho, P. Augusto and A. L. Gonalves, Mon. Not. Roy. Astron. Soc. 463 (2016) no. 3,2348

[28] C. C. Lin, L. Mestel, and F. H. Shu, Astrophys. J. 1421431 (1965)

[29] Y. B. Zel'dovich, Astron. Astrophys. 584 (1970)

[30] T. Harada, C.-M. Yoo, K. Kohri, K.-I. Nakao and S. Jhingan, Astrophys. J. 833, no. 1, 61 (2016)

[31] B. J. Carr, T. Tenkanen and V. Vaskonen, Phys. Rev. D 96 (2017) no.6, 063507

[32] F. Kühnel, and M. Sandstad, Phys. Rev. D 94063514 (2016)

[33] P. J. E. Peebles, Astrophys. J. 155, 393 (1969).

[34] P. Catelan and T. Theuns, Mon. Not. Roy. Astron. Soc. 282, 436 (1996)

[35] P. J. E. Peebles, "Principles of physical cosmology," Princeton, USA: Univ. Pr. (1993) 718 p

[36] J. c. Hwang, H. Noh and J. O. Gong, Astrophys. J. 752 (2012) 50

[37] A. G. Doroshkevich, Astrofizica 6581 (1970)

[38] D. H. Lyth, K. A. Malik and M. Sasaki, JCAP 0505 (2005) 004 
[39] M. Kopp, S. Hofmann, and J. Weller, Phys. Rev. D 83124025 (2011)

[40] P. J. E. Peebles, Large Scale Structure of the Universe, (Princeton Univ Press, Princeton, NJ, 1980) 